\title{
Effect of wind turbine wakes on wind-induced motions in wood-pole overhead lines
}

\author{
C. Maclver ${ }^{1}$, A. Cruden ${ }^{2}$, W. E. Leithead ${ }^{1}$ and M. P. Bertinat ${ }^{3}$ \\ 1 Department of Electronic \& Electrical Engineering, University of Strathclyde, 204 George St, Glasgow G1 1XW, UK \\ 2 Engineering Sciences, University of Southampton, Highfield, Southampton SO17 1BJ, UK \\ ${ }^{3}$ EA Technology Ltd, Capenhurst Technology Park, Capenhurst, Chester CH1 6ES, UK
}

\begin{abstract}
Wood-pole overhead conductor lines are known to be subject to different wind-induced motions, some which lead to conductor fatigue over long periods and others that can cause severe damage over short, isolated incidences. These lines are often located in rural areas, and, with the ever increasing deployment of onshore wind farms, the potential impact of the wind turbine wakes on these conductor motions is a matter of interest. Discussion of the potential effects of conductor fatigue on wood-pole overhead conductor lines is important as this could impact the planning, modelling and siting of onshore wind farms. This paper presents a literature analysis of both the mechanism by which different conductor motions are excited and wind turbine wake characteristics with specific interest at low levels akin to wood-pole height. The archival value of this paper is that experimental data from an active wind farm, at wood-pole overhead line heights, is analysed and discussed for the first time, and the results show that wake effects at these heights are likely to be minimal. However, turbulent buffeting in particular is cited as a problem of interest, and the potential effects on wood-pole motions are outlined. Copyright @ 2014 John Wiley \& Sons, Ltd.
\end{abstract}

KEYWORDS

aeolian vibration; galloping; buffeting; wake characteristics

Correspondence

C. Maclver, Department of Electronic \& Electrical Engineering, University of Strathclyde, 204 George St, Glasgow G1 1XW, UK.

E-mail: callum.maciver@strath.ac.uk

Received 19 December 2012; Revised 2 August 2013; Accepted 6 January 2014

\section{INTRODUCTION}

Wind-induced conductor motion is an issue which has been observed and studied for many years. Particular interest has been paid to the impact on large-scale high-voltage (HV) transmission networks, and the use of dampers to minimize the damaging effects of these motions is widespread on tower lines. Medium voltage (MV) wood-pole overhead lines (OHLs) are much more numerous, as shown in Table I, than large-scale tower lines and are therefore more likely to be sited in rural areas close to wind farms. They are also less critical lines and as such are less likely to make use of damping devices because of cost implications. The impact of wind turbines or wind farms and their resultant wakes on wood-pole OHLs, which stand at roughly $6 \mathrm{~m}$ height, has not been studied and so is the particular interest of this paper.

A thorough literature review of the different types of conductor motions that are known to exist and their impact has been undertaken and is presented in this paper. Further to this, an investigation into the characteristics of wind turbine wakes and the manner in which they propagate with particular reference to wood-pole height has been undertaken, supplemented by experimental measurements of wind data at $6 \mathrm{~m}$ taken from an operational wind farm in the UK. A review of the results and a discussion of the reliability of the data are presented. Finally, assertions have been made as to the probable wake impact on wood-pole lines as well as the worst case impact.

\section{WIND-INDUCED CONDUCTOR MOTIONS}

There are four main types of wind-induced motions which affect OHL conductors: aeolian vibration, galloping, buffeting and wake-induced galloping. The last applies only to bundled conductors, which do not usually feature on wood-pole 
Table I. Typical parameters of MV and HV lines. ${ }^{1-3}$

\begin{tabular}{lccc}
\hline & Typical span length $(\mathrm{m})$ & Typical conductor ground clearance $(\mathrm{m})$ & Estimated number of circuit km in UK (2002) \\
\hline MV OHL & $80-140$ & 6 & 335,886 \\
HV tower line & 360 & $12-50$ & 22,442 \\
\hline
\end{tabular}

OHLs and so is not considered in this analysis. The three remaining conductor motions are outlined in the succeeding text, as well as a brief overview of the main characteristics of the conductors in question.

\subsection{Aeolian vibrations}

Also known as vortex-induced vibrations, aeolian vibrations occur in wind speeds of around $1-7 \mathrm{~m} \mathrm{~s}^{-1}$ and generally excite frequencies in the range of $3-150 \mathrm{~Hz}^{4}$ They tend to induce low amplitudes of vibration in conductor lines, which can lead to fatigue damage, especially at support points or points where external hardware is connected to the line. A smooth constant flow passing over the length of a cylinder will cause vortices to be shed alternately from its top and bottom surfaces at a constant frequency. These Von Karman vortex streets shown in Figure 1 cause alternating pressure loads, which act in a perpendicular direction to the incoming flow.

The frequency at which the vortices are shed is referred to as the vortex shedding frequency or Strouhal frequency and is dependent on the wind velocity, $\mathrm{V}$ in $\mathrm{m} \mathrm{s}^{-1}$, and the diameter of the conductor, $\mathrm{D}$ in $\mathrm{m}$, as defined in equation (1). ${ }^{4}$

$$
F_{S}=N_{S} \frac{V}{D}
$$

$\mathrm{N}_{\mathrm{s}}$ is a constant known as the Strouhal number and takes a typical value in the order of 0.185 for OHL conductors. ${ }^{4,6}$ For a flexibly mounted conductor span in a steady wind, these effects can result in an oscillatory motion. The amplitude of vibration experienced by a conductor in a cross flow is minimal, however, until the Strouhal frequency closely matches a natural mode of the conductor. The nth order natural modes of overhead conductors can be found using equation (2), where $\mathrm{T}$ is the tension $(\mathrm{N}), \mathrm{w}$ is the mass per unit length $\left(\mathrm{kg} \mathrm{m}^{-1}\right), 1$ is the span length of the conductor $(\mathrm{m})$ and $\mathrm{n}$ is the modal order such that $n=1$ gives the fundamental frequency of the conductor. ${ }^{6}$ Typical values for the fundamental frequency of conductor lines are discussed in Annex 1.3 but tend to be of very low magnitude in the order of $0.1-0.3 \mathrm{~Hz}$ for $\mathrm{HV}$ transmission lines. ${ }^{4}$ It is known that excitation can occur over a large frequency range of around $3-150 \mathrm{~Hz}^{4}$ so the number of steady wind speeds capable of exciting an nth order natural frequency is not confined to one or two specific values but is extensive.

$$
f_{n}=n \sqrt{\frac{T}{w}} \frac{1}{2 L}
$$

Once a natural mode is excited, the motion is enhanced and the alternating pressure loads excite a periodic oscillatory motion in the conductor whereby vibration amplitudes of up to one diameter can occur. The vibrations are, however, self limited to this maximum as beyond this, the shed vortices no longer maintain the stable alternating pattern of Figure $1 .{ }^{7}$

If the induced oscillations are sufficient, a frequency lock-in phenomenon can occur such that the frequency of the shed vortices is determined by the oscillations and not by the Strouhal frequency determined by the incoming wind speed. This can persist for variations in wind speed up to $\pm 10 \% .{ }^{4}$ This means that Aeolian vibrations are possible even in fluctuating flows, and some reports suggest that vibration amplitudes will build up slowly to a steady maximum over time scales in the order of minutes for a turbulent wind providing there is a steady mean wind speed. ${ }^{8}$

Real turbulent winds however are such that the mean velocity is not fixed, and wind variations of more than $10 \%$ will be occurring most of the time such that any lock-in modes will be broken quickly. Turbulence will also act to break up the vortices as they are shed from the conductor, thus reducing the energy imparted to the conductor. Turbulence is therefore generally considered to reduce the impact of vortex-induced vibrations. ${ }^{6}$

If a constant, steady wind field is considered, then the aeolian vibrations that are induced can be modelled simply as a number of standing waves with a specific loop length. The number of standing waves is given by $n$ in equation (2) and is

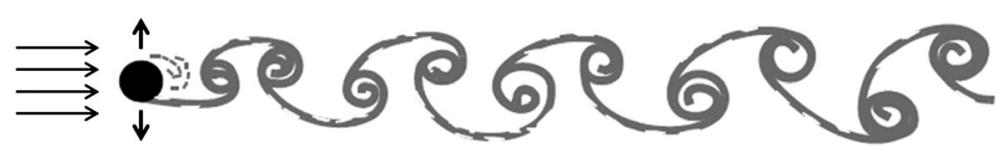

Figure 1. Motion-inducing vortices shed by a cylinder in a smooth cross flow. ${ }^{5}$ 
the same as the order of mode that is being excited. As the wind speed and Strouhal frequency increase such that higher order modes are excited then so too does the number of standing waves on the line. The amplitude of vibration however is likely to decrease as the wind speed and mode numbers increases up to the point where there is not enough energy in the wind to sustain the high frequency oscillations. ${ }^{7}$ In reality however a conductor will be subject to a varying wind field and therefore numerous vibrational frequencies are likely to be excited simultaneously. Each vibrational frequency will pulse through the conductor and combine to produce a resultant beating pattern (Figure 2) as opposed to a purely sinusoidal vibration pattern of a single frequency.

\subsubsection{Fatigue damage due to aeolian vibration}

Aeolian vibrations are widely recognized as the number one contributor to fatigue failures in conductors. The failures are almost exclusively found to occur at points on the line where it is constrained against transverse motion such as at support locations. Fatigue damage has been observed on all conductor types and as such there are design standards and damping equipment that are adhered to and implemented to minimize the effect of aeolian vibrations. The use of dampers however is mainly restricted to large-scale HV transmission lines, which are more critical to the operation of the grid.

Fatigue damage is a direct result of the cyclic bending induced by aeolian vibrations at support points although the damage is actually mostly associated with fretting fatigue as opposed to simple bending stress fatigue. This is a result of the stranded nature of conductors whereby the cyclic bending leads to cyclic microslip and so rubbing and wear of the strand surfaces where they are in contact with external supports or each other. ${ }^{4}$ In general, an increase in the amplitude of bending will give rise to increased levels of fretting, meaning fatigue damage will occur more quickly. Like many materials, conductors have been found to possess an endurance limit in terms of fatigue analysis, that is, a stress limit below which the vibrations can be endured indefinitely and above which fatigue will begin to occur. This level will be specific to the conductor clamp combination being investigated.

Aeolian vibration activity is known to increase with conductor tension due to a reduction in self damping and is also affected by the level of turbulence in the area. As such, a Cigré task force ${ }^{9}$ has developed a set of recommended maximum design tensions on the basis of four levels of terrain, each associated with a different turbulence level. The limiting values are based on the $\mathrm{H} / \mathrm{W}$ ratio or the ratio of initial design tension, $\mathrm{H}(\mathrm{kg})$, to the mass per unit length, $\mathrm{W}\left(\mathrm{kg} \mathrm{m}^{-1}\right)$, of the conductor. The allowable safe maximum for this ratio increases as the terrain-induced turbulence increases. For most wind turbine sites, the level of turbulence would fall into either the second or third terrain categories describing undulating terrain with significant turbulence present. The ratios required for these two terrain categories are 1125 and 1225 , respectively, so these should not be exceeded to minimize the effects of aeolian vibrations. The values are based on the initial design tension referred to the mean temperature of the coldest month of the year and so are closely related to the maximum tensions that the conductor is likely to experience.

\subsection{Conductor galloping}

Galloping is a well-known phenomenon and can lead to severe damage to overhead power lines. It almost always occurs because of ice building up around the conductor creating an asymmetry, which can, under the correct conditions, lead to divergent oscillations. These low frequency and high-amplitude oscillations tend to primarily act vertically and are caused by moderately strong crosswinds of around $7-20 \mathrm{~m} \mathrm{~s}^{-1}$. The implications of conductor galloping can be very serious both through physical damage to the line and the potential for conductor clashing and electrical faults.

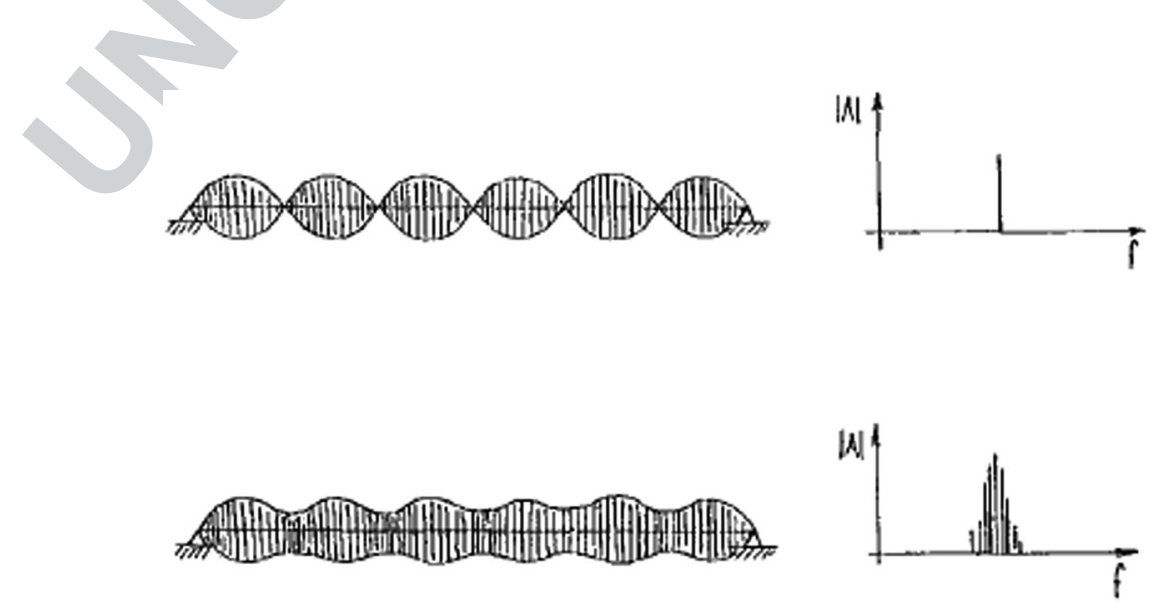

Figure 2. Beating pattern of a conductor in turbulent flow. ${ }^{8}$ 
A perfectly cylindrical conductor will not gallop under any wind-induced conditions. However, there is such an occurrence as bare-wire galloping whereby uniced conductors with a large number of outer strands can experience, under very specific conditions, lift forces due to differences in the apparent roughness of the top and bottom surfaces of the conductor. ${ }^{4}$ This however is a very rare phenomenon and as such will not be considered further.

Galloping occurs as a result of an instability related to the lift and drag components generated on an iced conductor that is asymmetrical with respect to the apparent wind speed it is subject to. Classical theory laid out by Den Hartog ${ }^{4,10}$ explains the mechanism by which galloping instability can occur. Any conductor is subject to a drag force, which acts in the same direction as the relative wind velocity, $\mathrm{V}_{\mathrm{r}}$. If an asymmetry is present in the conductor/ice combination with respect to $V_{r}$, then a lift force will also be present (Figure 3). This always acts at right angles to the relative velocity vector, but its sign and magnitude depend on the nature of the asymmetry that $\mathrm{V}_{\mathrm{r}}$ experiences. The lift force cannot be predicted by airfoil theory because of the turbulence in the wake of conductor type structures invalidating the potential flow assumption.

A vertical motion of the conductor itself will modulate the apparent wind vector, $\mathrm{V}_{\mathrm{r}}$, and give rise to different angles of attack. As the angle of attack of the apparent wind vector changes, then so too does the asymmetry of the conductor with respect to this. Thus, the lift vector will vary as a function of this angle of attack, and an example plot of this can be seen in Figure 4.

If a conductor is given an upwards vertical velocity, the resultant relative wind direction and particular angle of attack, $\beta$, can be seen in Figure 5. Also shown are the lift and drag forces that the iced conductor will be subject to, and it is clear that a component of the lift force will act in an upwards direction and hence aid the upwards motion. If the motion given to the conductor is downwards, then the lift force will have a downwards component, which acts to aid the downwards motion. This shows that for certain iced conditions and over a certain range of $\beta$ values, an oscillating conductor can be subject to a lift force that provides negative damping.

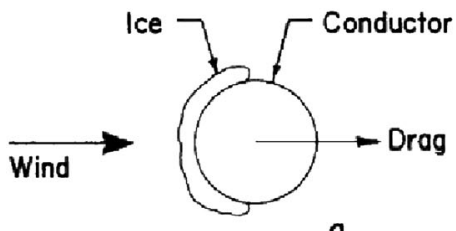

a.

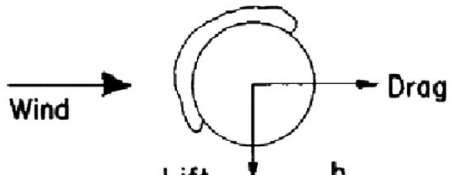

Lift

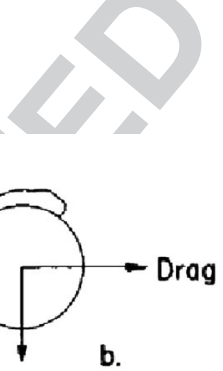

b.

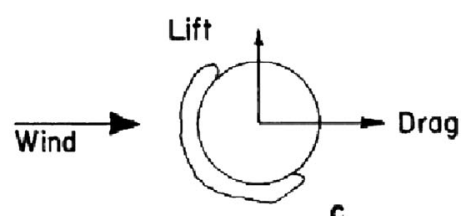

c.

Figure 3. Lift and drag on three different iced conductors. ${ }^{4}$

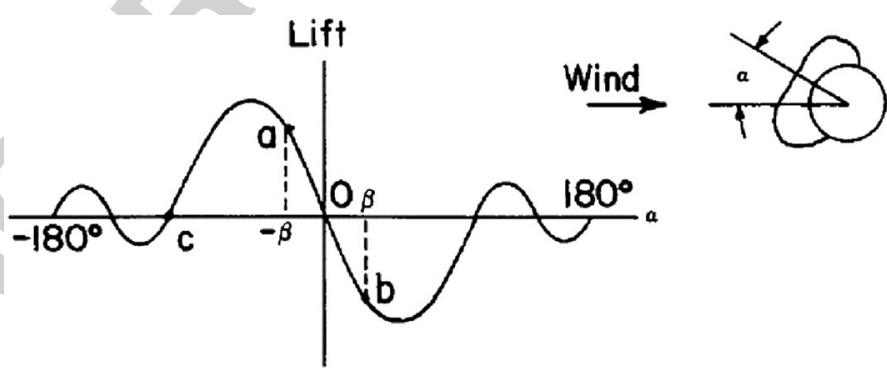

Figure 4. Lift variation with angle of attack. ${ }^{4}$
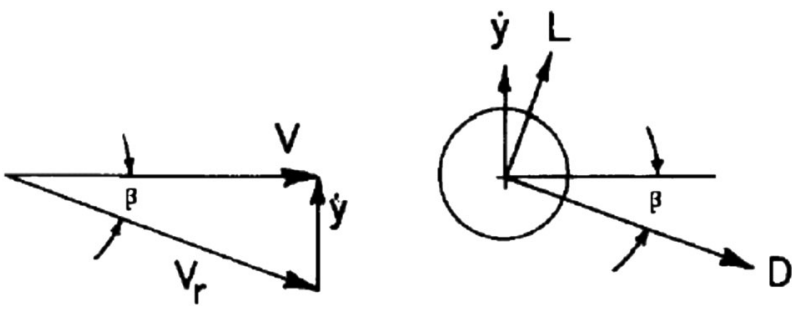

Figure 5. Lift and drag force on a vertically moving iced conductor. ${ }^{4}$ 
This negative damping is counteracted by the positive damping that is associated with the drag force as well as the inherent self damping of the conductor. This self damping however is very low for conductors at the low frequencies at which galloping events occur. If the changes in angle attack are limited to the straight line region between a and $b$ in Figure 4, then the lift can be given by equation (3).

$$
L=\frac{d L}{d \alpha} \cdot \beta
$$

Thus, the negative damping component depends on the rate of change of lift, and from this, the well-known Glauert-Den Hartog criterion can be derived such that for galloping instability to occur,

$$
\frac{d L}{d \alpha}+D<0
$$

must be satisfied. That is, the slope of the lift curve in the specified region must be more negative than the total drag, D, is positive. This means that for any given iced conductor, it is possible to compare the derivative of lift and drag parameters as a function of angle of attack to identify the zones of angle of attack, where the Den Hartog criteria is satisfied and galloping instability can occur. For real iced conductors, these zones are likely to be relatively small and are dependent on the nature of the icing in question. ${ }^{4}$ If the angle of attack enters a Den Hartog zone, however, then the net damping is negative and so small vertical perturbations can diverge and lead to high amplitude and low frequency conductor galloping.

Galloping may often begin as relatively small-sized travelling waves, which pulse through the line and can gradually grow in size and amplitude until large low-order standing wave oscillations are incident over the whole length of the conductor. Galloping incidences generally result in first, second or third-order mode shapes and generally will not occur at frequencies of above $3 \mathrm{~Hz} .^{4}$

There are restrictions to the level of divergence possible, and reference to Figure 4 shows that large changes in angle of attack will move the conductor out of the zone that gives the large negative slope required for instability. Thus, the amplitude of vibration is a limited parameter. In reality, galloping does not often occur as a purely vertical motion but also has a torsional aspect, which creates an elliptical galloping path.

\subsubsection{Damage due to galloping}

Galloping events on short-span distribution lines can lead to conductor oscillations of up to four times the size of the natural sag of the conductor. ${ }^{4}$ Naturally, such severe oscillations exert large dynamic loads on supporting structures, which have been known to cause failure in cross-arms and even loosen the ground support of wood poles themselves. Less violent incidents may simply add to fatigue damage of lines or related structures.

If oscillations are such that flashover occurs between phases, then damage of the conductors can be caused by arcing though the faults are usually brief enough that physical conductor damage of this sort is not a common issue. The related outages however can be costly in terms of lost revenue. In fact, any fault, mechanical or electrical, will have a financial cost associated with it, and these can be quite severe depending on the magnitude of damage, time required for repair and the number of customers affected by any outages.

As a result of this, protection methods against galloping have been employed and generally fall into three categories. The first of these is the removal or prevention of ice formation on the conductor. This has been attempted by several means of electrical heating above and beyond normal loading of the lines. Second, dampers can be used to interfere with either the energy balance associated with galloping (similar to how Stockbridge dampers work to reduce aeolian vibrations) or with the torsional motion and so restrict the build-up of the large amplitudes of vibration. Lastly, design features such as increased phase clearance to minimize flashover potential or interphase ties, which control the modes of galloping, can be included as protection. As with aeolian vibration, these devices or solutions are most likely to be concentrated on large-scale transmission lines as opposed to rural wood-pole lines because of the economics involved.

\subsection{Turbulent buffeting}

In a turbulent flow field, as might well be expected in locations of interest for this investigation, a third wind-induced phenomenon known as turbulent buffeting may be the most important consideration. Buffeting is attributable to variations in the drag forces induced on a conductor by a turbulent wind field.

Any structure will experience a drag force in an incident wind. This is oriented in the same direction as the wind for a still conductor or in the direction of relative wind speed for a moving conductor (Figure 3). The drag force, $F_{D}(N)$, on a circular cylinder is given by equation (4) where $\rho$, is the air density $\left(\mathrm{kg} \mathrm{m}^{-3}\right), \mathrm{d}$, the conductor diameter $(\mathrm{m}), \mathrm{C}_{\mathrm{D}}$, the drag coefficient and $\mathrm{V}_{\mathrm{r}}$, the relative velocity $\left(\mathrm{m} \mathrm{s}^{-1}\right)$.

$$
F_{D}=\frac{1}{2} C_{D} \rho d V_{r}^{2}
$$


The drag coefficient is dependent on the Reynolds number, Re, which in turn depends on velocity, while $C_{D}$ can be considered constant up to a critical Re, where it will drop sharply to a new lower value. Changes in wind velocity and/ or wind direction, due to turbulence, can induce sharply varying drag forces given the second-order relationship to velocity as will transitions over the critical Re value. In a turbulent wind, these variations will occur randomly in the spanwise direction and so impose dynamic movement upon the conductor in all directions.

Such forced dynamic motions will inevitably lead to fatigue loading at conductor support points, which may have a similar impact on the line as aeolian vibrations (Section 2.1.1). In fact, it has been reported, on the basis of experimental evidence from various sources, that seemingly random aeolian vibrations in turbulent winds are in fact turbulent buffeting as opposed to vortex-induced vibrations. ${ }^{11}$ In strong, stormy conditions, the forced motions may be very large and have effects akin to those of conductor galloping. ${ }^{4}$ Figure 6 shows an example of buffeting of a long span conductor at $30 \mathrm{~m} \mathrm{~s}^{-1}$ wind speed with several metres movement in the flow and vertical directions (this is a coupled motion) with oscillations in both directions in the order of a metre. Such movement would induce large loads on supporting structures, and even when scaling down to lower winds and shorter span length, it is evident that conductor fatigue may well be an issue in turbulent winds.

Possibly of significant importance is the fact that the buffeting response of a structure is amplified around its natural frequency. ${ }^{12}$ The buffeting response will therefore be sensitive to the frequency content of the wind. This is an important point as a wind turbine wake may contain frequency content close to the natural frequency of a conductor or its second or third modes as will be explored in subsequent sections.

\section{WOOD-POLE CONDUCTOR CHARACTERISTICS}

Much of the work relating to wind-induced conductor motions has focused on HV transmission lines, and one of the motivating factors behind this research is to investigate how far this analysis can be applied to the lower standing, shorter span sections associated with MV wood-pole lines.

There are numerous types of conductor used in the UK, but the three most common are all-aluminium alloy conductor (AAAC), aluminium conductor steel reinforced (ACSR) and all-aluminium conductor. ${ }^{1}$ With reference to current design standards given by distribution network operators, it appears that AAAC-type conductors are currently the most favoured although ACSR have been popular in the recent past. ${ }^{13,14}$ Each type has specific operational characteristics, meaning different conductor types may be preferred for different reasons. Where strength/weight ratio is the main concern, then AAAC is likely to be chosen, whereas ACSR was popular because of its strength allowing for longer span sections and therefore lower overall costs. ${ }^{1}$

For analysis of all the wind-induced motions, an essential parameter is the conductor natural frequency. This can be used to calculate the array of wind speed values that will be most capable of exciting aeolian vibrations but is also an important aspect for both galloping and buffeting motions as has been explained. For a typical UK wood-pole distribution line, the span lengths are typically in the range of $80-140 \mathrm{~m} .{ }^{1}$ Using these figures and the maximum safe design tension limits outlined by Cigré, ${ }^{9}$ the natural frequency of certain conductors can be predicted. From equation (5), the natural frequency of a conductor given in terms of the H/W ratio, it is clear that the natural frequency is dependent on the tension of the conductor, and this is by no means a constant being affected by both temperature changes in the short term and conductor creep in the long term.

$$
f_{n}=\sqrt{\frac{H g}{w}} \frac{1}{2 L}
$$

The fact that newly installed cables will be designed not to exceed these tensions for the vast majority of time means that using the $\mathrm{H} / \mathrm{W}$ ratio in the aforementioned formula for a given cable and a given terrain will yield what can be considered to be an upper limit on the natural frequency of the conductor over a period. For older conductors of this type, the previous
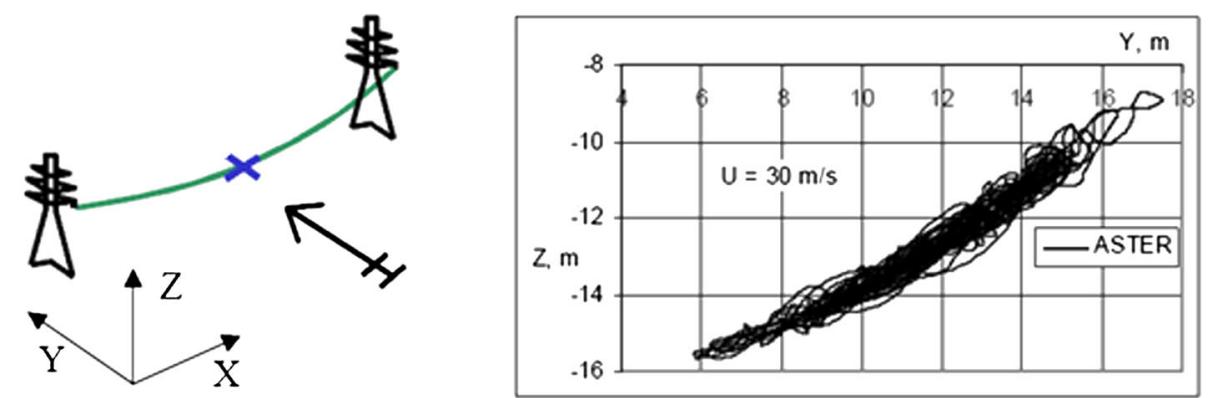

Figure 6. Conductor motion of $500 \mathrm{~m}$ span aster cable due to buffeting in $30 \mathrm{~m} \mathrm{~s}^{-1}$ wind. 
industry design standard required that a nominal design stress value of $18 \%$ of rated tensile strength be adhered to. ${ }^{1}$ This limited the tension of the conductor at the temperature for which it is subject to for the longest period. For conductors subject to the latter criteria, the \% rated tensile strength value can be used to work out an upper limit on the everyday natural frequency of a conductor.

At high summer UK temperatures, the still air tension of AAAC conductors can reduce by as much as $50 \%$ on the design tensions for the coldest periods. ${ }^{1}$ Thus, for recent or new installations, a range of likely conductor natural frequencies can be calculated from equation (5), using lower limit H/W ratios, which are half that of the upper limit values. If the new standards are adhered to, the natural frequencies calculated should be common to all conductor types as the tension is varied to compensate for the differences in weight per unit length of each conductor. Table II shows the values calculated for four different span lengths and two different turbulent terrain categories.

It should be noted that the thermal expansion coefficient of AAAC is actually higher than that of ACSR conductors so the values in Table II are unlikely to be fully indicative of all conductor types. However, they do show the general figures and trends that need to be considered. The first thing of note is that the natural frequency varies with the inverse of the span length, and so, long-span transmission lines are likely to have lower natural frequencies than short-span distribution lines. This is an important outcome as it means that wood-pole OHLs may be less susceptible to aeolian vibrations than long-span transmission lines yet more susceptible to the frequency content of wind turbine wakes, increasing the risk of largeamplitude buffeting response.

\section{WAKE EXPANSION AND PROPAGATION}

\subsection{General wake analysis}

To evaluate the risk posed by wind turbine wakes to OHLs at heights of around $6 \mathrm{~m}$, it is necessary to investigate how the wake of a wind turbine will expand and propagate downstream in turbulent wind. Of particular interest are the lower extent of the wake expansion and the characteristics of the flow field within the wake.

Actuator disc theory (Figure 7) illustrates the high-level features of a wind turbine wake showing how the wind velocity reduces as it approaches the rotor in turn leading to an expansion of the stream tube as dictated by conservation of mass flow rate. These two features continue past the rotor until the wake is fully expanded and the velocity deficit has peaked.

Table II. Natural frequency ranges expected for different span lengths and turbulence.

\begin{tabular}{lcccr}
\hline & & \multicolumn{3}{c}{ Expected natural frequency range $(\mathrm{Hz})$} \\
\cline { 2 - 5 } Span length $(\mathrm{m})$ & 80 & 100 & 120 & 140 \\
\hline $15 \%$ turbulence & $0.46-0.66$ & $0.37-0.53$ & $0.31-0.44$ & $0.27-0.38$ \\
$22 \%$ turbulence & $0.48-0.69$ & $0.39-0.55$ & $0.32-0.46$ & $0.28-0.39$ \\
\hline
\end{tabular}

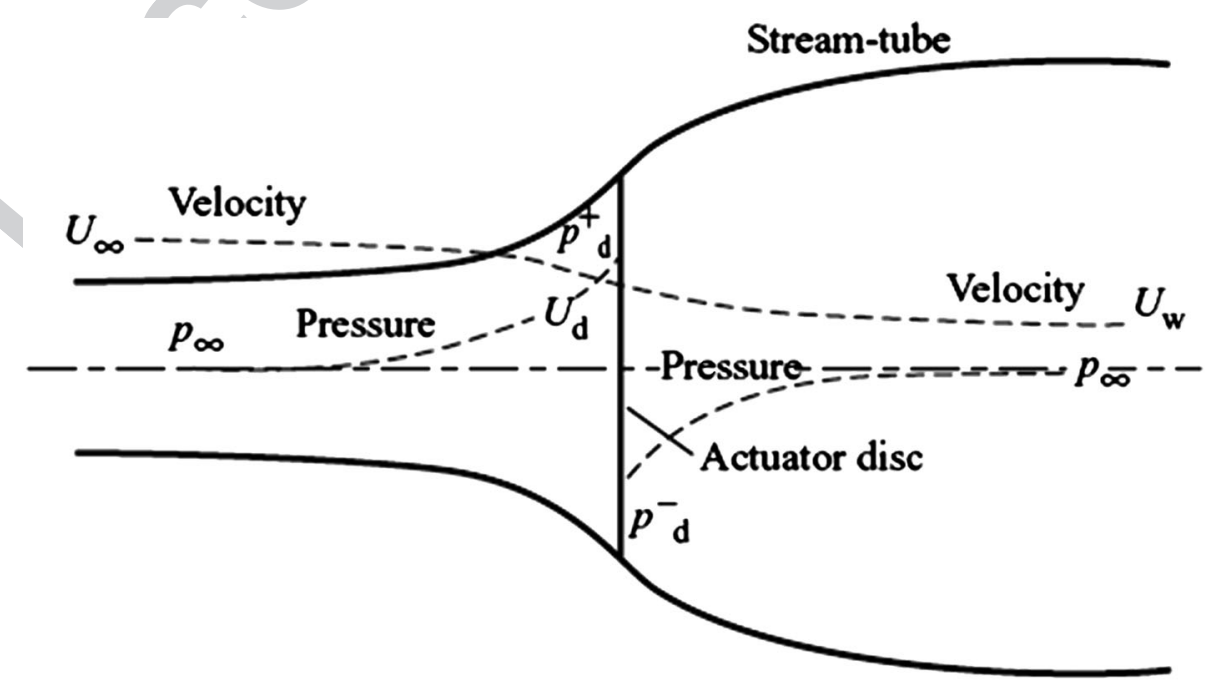

Figure 7. Actuator disc theory stream tube model. 
A simple calculation for the extent of the wake expansion can be carried out using the principle of mass flow rate conservation equation (7) and assuming a constant axial flow induction factor, a, of one-third. The induction factor is a measure of the blockage the rotor presents to the incoming flow, and one-third is the optimum value for maximum power extraction. Using equation (6), the reduced velocity in the wake, $\mathrm{U}_{\mathrm{w}}$, is found in terms of the velocity at the rotor, $\mathrm{U}_{\mathrm{D}}$, allowing equation (7) to be solved for $A_{w}$, the expanded wake area, in terms of the rotor area, $A_{D}$, which will be a known quantity.

$$
\begin{gathered}
U_{w}=(1-a) U_{D} \\
\rho A_{w} U_{w}=\rho A_{D} U_{D}
\end{gathered}
$$

This basic calculation predicts a final wake area expansion of 50\% compared with the rotor area, which corresponds to an increase in the radius of the wake of $22.5 \%$. If the axial flow induction factor is lower than this ideal value of $\frac{1}{3}$ as in the case of pitched blades, for example, then the expansion will be smaller than this. This is broadly consistent with other studies ${ }^{15}$ that have sought to investigate the true expansion of wind turbine wakes although some, using other methods, have predicted smaller expansions in the order of $10 \% .^{16}$

In reality, wind turbine wakes are influenced heavily by interaction with the free flow outside the wake, and a more realistic depiction of the wake development is given in Figure 8. Wakes are considered as being comprised of different regions, the first of which is the near wake. This is the area beginning immediately after the rotor, where the axial velocity is still reducing. The near wake is characterized by a strong velocity deficit in the centre of the wake region encircled by a shear layer generated by the difference between the velocity inside the wake and the free stream velocity. This shear layer leads to distinct peaks in turbulence intensity around the wake perimeter, the largest of which are in the upper half of the wake due to the additive influence of ambient shear. Turbulent eddies are formed in this region, and as the wake propagates downstream, the shear layer expands because of increased turbulent mixing until the wake centreline is reached. This marks the end of the near wake. The strong characteristics of the near wake are generally considered to be present within the first two rotor diameters (2D) downstream, with the initial expansion occurring over the first $1 \mathrm{D}$ downstream ${ }^{17}$ and full wake expansion being complete by $2.25 \mathrm{D}$ using comparisons with experiments. ${ }^{18}$ In keeping with this, ${ }^{19}$ Ainslie predicts maximum velocity deficit between $1-2 \mathrm{D}$ downstream $a^{20}$ assert that the transition to the far-wake state will occur between 2-5D downstream.

The far wake can be modelled as having an approximately axisymmetric velocity deficit profile with Gaussian distribution. In fact, the presence of the ground means that the lower half of the wake is subject to less turbulent mixing, meaning that the peak velocity deficit occurs in the lower half of the wake. The far-wake field will continue to expand as turbulence encourages further mixing with the free stream, which will eventually bring the velocity deficit back to zero and end the presence of the wake. Turbulence intensity in the far wake no longer has distinct peaks around the outer perimeter of the wake but will also form an approximately Gaussian profile, which, as in the near wake, will be highest in the upper half of the wake. ${ }^{15}$ The level of both the turbulent intensity and velocity deficit will diminish as the wake propagates downstream, and wind farm developers have estimated that wake effects on energy capture will be sufficiently small after $7 \mathrm{D}^{17}$ although as Figure 9 illustrates, there are still wake effects present up to 10D and beyond with increased turbulence intensity effects found to propagate further than velocity deficit effects (measured as the ratio of measured velocity, $\mathrm{U}$, to free stream velocity, $\mathrm{U}_{\mathrm{o}}$ ).

A further aspect of interest, not yet explored, is the fact that turbine blades shed tip vortices that propagate downstream in helical paths, and it is these vortices which make up the shear layer, which initially separates the slow moving, expanding wake from the free stream flow. ${ }^{15}$ The tip vortices will develop at a frequency of three times the rotational frequency of the

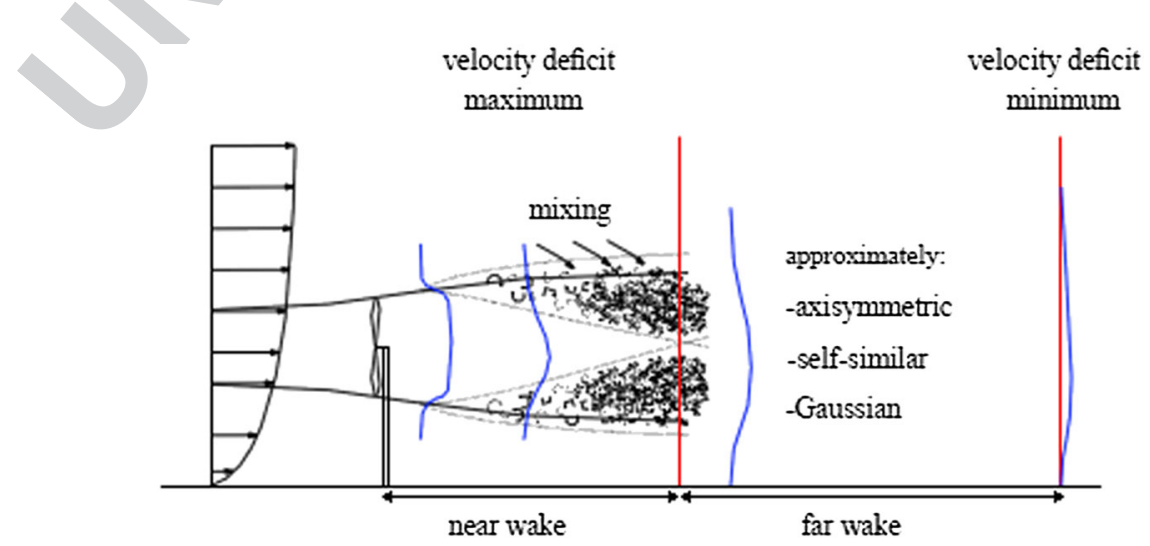

Figure 8. Wake development showing shear layer growth and example velocity deficit. ${ }^{15}$ 

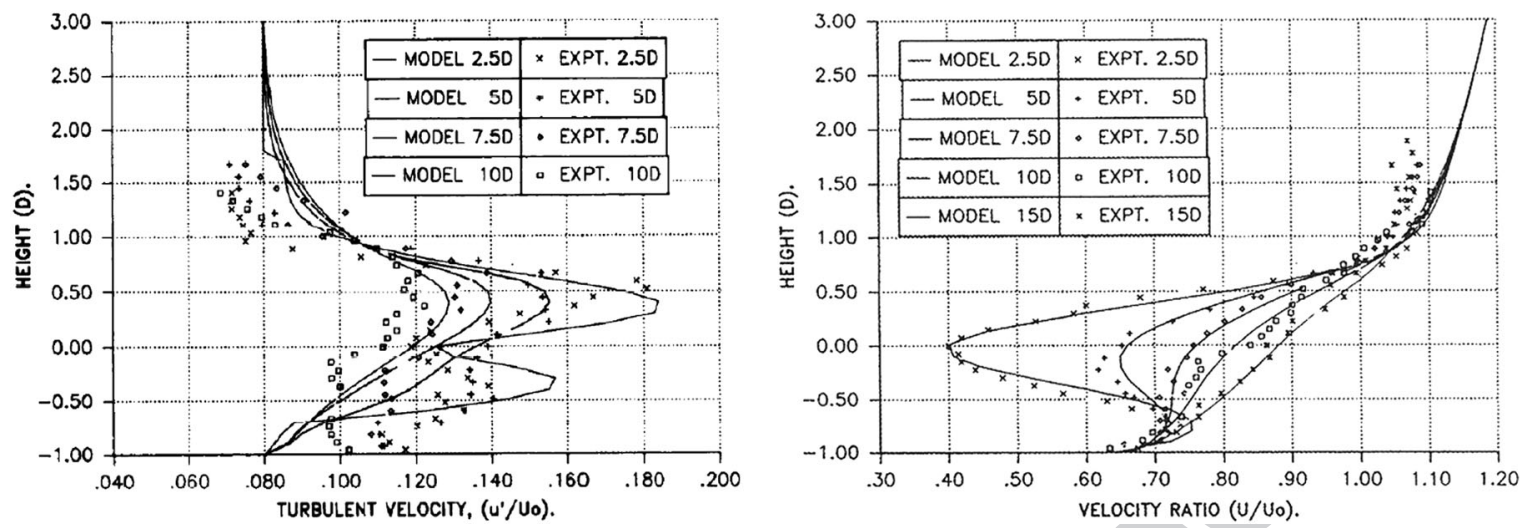

Figure 9. Turbulence intensity and reduced velocity as a function of downstream distance. ${ }^{21}$

turbine for a three-bladed machine. Simulations by Troldborg et al. ${ }^{22,23}$ have modelled the propagation of these vortices in a turbulent flow as shown in Figures 10 and 11. It can be seen that the vortices in this model are still distinguishable up to around 3D downstream after which the wake has become fully turbulent. This is broadly consistent with Ainslie, ${ }^{19}$ who suggests the presence of tip vortices up to 4D downstream.

\subsection{Wake impact at wood-pole height}

With reference to the aforementioned analysis, an estimation of the probable wake effects at wood-pole OHL height can be made. It must first be noted that the size, height and distance from any OHL of the turbines in question are important parameters. If the hub height and rotor radius are known for any turbine, the predicted wake expansion, assuming an ideal axial induction factor, can be calculated from equations (6) and (7). Table III outlines the minimum height that the wakes will drop to after initial expansion 2-3D downstream for some different example sites in the UK.

The initial expansion of turbine wakes evidently does not reach as low as $6 \mathrm{~m}$ in the vast majority of cases. In fact, when considering commercial projects, only the smallest turbines will be low enough to have an initial expansion anywhere close to $6 \mathrm{~m}$ from ground level, assuming reasonably flat ground. As turbines develop in size and scale, the hub heights that they operate at are increasingly high, bringing the wake expansion further away from wood-pole height.

a)

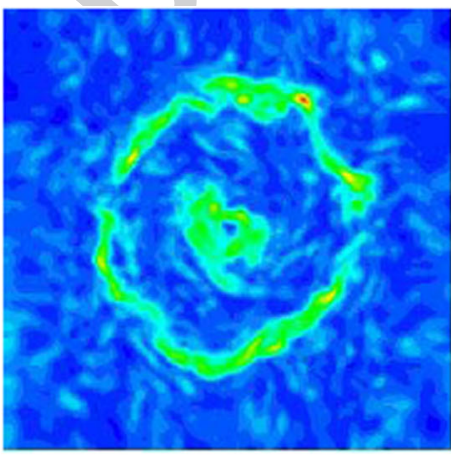

b)

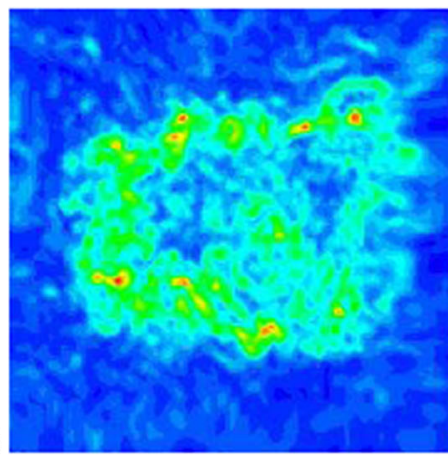

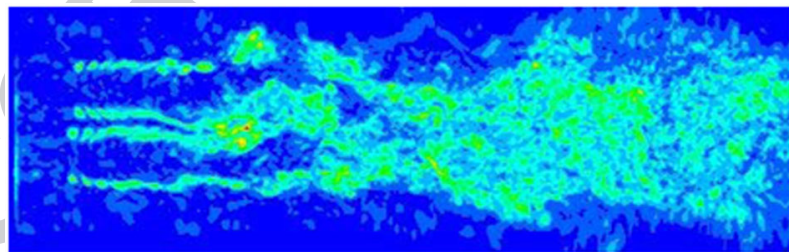

Figure 10. Wake development for turbulent inflow at $U=10 \mathrm{~m} \mathrm{~s}^{-1}{ }^{23}$

Figure 11. Planar vorticity contours at (a) $1 \mathrm{D}$, (b) $3 \mathrm{D}$ and (c) $5 \mathrm{D}$ downstream of the turbine. ${ }^{23}$ 
Table III. Wake expansion at some example UK sites.

\begin{tabular}{lccccc}
\hline UK site & Turbine rating $(\mathrm{MW})$ & Rotor radius $(\mathrm{m})$ & Hub height $(\mathrm{m})$ & Rotor clearance to ground $(\mathrm{m})$ & Wake clearance to ground $(\mathrm{m})$ \\
\hline Coal Clough & 0.4 & 17 & 30 & 13 & 9.2 \\
Cruach Mhor & 0.85 & 26 & 45 & 19 & 13.2 \\
Whitelee & 2.3 & 50.5 & 100 & 49.5 & 38.2 \\
\hline
\end{tabular}

Indeed, the issue with potential wind-induced motions of wood-pole OHLs is actually growing in concern, certainly in the UK, where recent planning applications have proposed the use of wood-pole $132 \mathrm{kV}$ OHLs of between 10.5 and $15 \mathrm{~m}$ in height, compared with conventional metal pylon transmission lines of typically up to $35 \mathrm{~m}$ height, to minimize visual impact. Indeed, a new $132 \mathrm{kV}$ single-circuit wood pole OHL, is proposed to originate from within the Llandinam wind farm to Welshpool substation in Wales.* This wind farm is the subject of an application to 'repower' the site by removing the 103 existing Mitsubushi $300 \mathrm{~kW}$ turbines installed in 1992 to 34 new larger Siemens turbines, type SWT-2.3-82 (2.3 MW, $82 \mathrm{~m}$ Q1 blade diameter). The proposed OHL will be within $100 \mathrm{~m}$ from the wind farm, ${ }^{\dagger}$ that is, $\angle 2 \mathrm{D}$ from the proposed repowering turbines. Hence, this paper describes an issue that is not only relevant to existing onshore wind farms but both planned new onshore wind farms and wind farms seeking to be 'repowered'.

Despite the aforementioned findings, it is perfectly feasible that further turbulent mixing will expand a given wake such that effects will be seen as low as $6 \mathrm{~m}$ at some point downstream of the turbine. Given the findings of Section 3.1, however, Q2 vorticity effects are unlikely to still be present by the time a wake has reached this level. Figure 9 shows that turbulence intensity and velocity deficit effects may still be present although both the modelled and measured values show very little change over ambient conditions at the lower extremes of interest. The velocity deficit in particular at low levels appears to show very little change on ambient levels. The turbulence intensity, at least in the measured values, shows slightly larger deviations at low levels, indicating that increased turbulence intensity could be the main wake effect at wood-pole height. Even so, any increases apparent at these outer extremities of the wake will be much smaller than the peak increases seen inside the main wake body.

\section{ANALYSIS OF EXPERIMENTAL DATA ${ }^{\ddagger}$}

\subsection{Test set-up procedure}

Experimental data was recorded at an operational wind farm on four separate days and can conveniently split into two sessions. Session 1 data was obtained in three sets of tests, taken on three separate days using three anemometers all mounted at the same height and all capable of recording only once per second. Session 2 data was obtained from a fourth day of tests, with the two downstream anemometers mounted at different heights and the higher one capable of recording four times per second. The cut-in speed for the Vestas WD34 turbines ${ }^{\S}$ monitored at Coal Q3 Clough is $5 \mathrm{~m} \mathrm{~s}^{-1}$.

\subsubsection{Test set-up: session 1}

Experimental data taken from an operational wind farm was analysed to supplement the findings of the previous work outlined in Section 4. In Session 1, three anemometer/data logger test sets were used to take wind measurements at $6 \mathrm{~m}$ height at different locations around the vicinity of a single operating turbine. The layout of the anemometers for each of the tests is described in Table IV and is shown schematically for day 2 in Figure 12. (Days 1 and 3 were similar but with different orientations relative to the wall to maintain fixed orientation relative to the wind.)

The maximum sampling rate achievable was $1 \mathrm{~Hz}$, and as such, a $1 \mathrm{~s}$ wind data was recorded. A reference test set was placed 1D upstream of the centre of the turbine and remained in place for the duration of the tests with the other two test sets moved to centre and off centre locations 1D, 2D and 3D downstream over a number of different tests. Results were accrued on three separate days.

\footnotetext{
*http://www.spenergynetworks.co.uk/serving_our_customers/consultation_llandinam.asp? NavID=1\&SubNavID=3\&SubSubNavID=7 ${ }^{\dagger}$ http://www.scottishpowerrenewables.com/userfiles/file/Llandinam\%20Repowering_English_Non\%20Technical\%20Summary.pdf

${ }^{\ddagger}$ Data gathering was funded by the Strategic Technology Programme, which is managed by EA Technology, and was carried out at the Coal Clough wind farm near Burnley, Lancashire, by kind permission of Scottish Power Renewables.

${ }^{\S}$ http://users.auth.gr/ karapant/tdk/Publications/files/Balouktsis_et_al_2002.pdf
} 
Table IV. Anemometer/logger positions and timings for each test (day 2).

\begin{tabular}{|c|c|c|c|c|c|c|}
\hline Day 2 & Logger 1 & Logger 2 & Logger 3 & Start & End & Duration \\
\hline Test 2.1 & 1D upstream (approx), on axis & & & 08:59:33 & $12: 31: 56$ & 03:32 \\
\hline Test 2.2 & 1D upstream (approx), on axis & 1D downstream, on axis & & 09:10:19 & $10: 14: 21$ & 01:04 \\
\hline Test 2.3 & 1D upstream (approx), on axis & 1D downstream, on axis & $\begin{array}{l}\text { 1D upstream (approx), } \\
\text { D/2 off axis (NE) }\end{array}$ & $09: 21: 58$ & 09:37:23 & $00: 15$ \\
\hline Test 2.4 & 1D upstream (approx), on axis & 1D downstream, on axis & $\begin{array}{l}\text { 1D downstream, D/2 off } \\
\text { axis (SW) }\end{array}$ & $09: 43: 51$ & 09:57:20 & $00: 13$ \\
\hline Test 2.5 & 1D upstream (approx), on axis & 1D downstream, on axis & $\begin{array}{l}\text { 1D downstream, D/2 off } \\
\text { axis (NE) }\end{array}$ & 10:01:02 & 10:18:16 & $00: 17$ \\
\hline Test 2.6 & 1D upstream (approx), on axis & 2D downstream, on axis & & 10:18:17 & $11: 04: 47$ & $00: 46$ \\
\hline Test 2.7 & 1D upstream (approx), on axis & 2D downstream, on axis & $\begin{array}{l}\text { 2D downstream, D/2 off } \\
\text { axis (SW) }\end{array}$ & 10:28:41 & 10:42:30 & $00: 13$ \\
\hline Test 2.8 & 1D upstream (approx), on axis & 2D downstream, on axis & $\begin{array}{l}\text { 2D downstream, D/2 off } \\
\text { axis (NE) }\end{array}$ & $10: 46: 13$ & 11:04:06 & $00: 17$ \\
\hline Test 2.9 & 1D upstream (approx), on axis & 3D downstream, on axis & & $11: 07: 48$ & $12: 18: 34$ & $01: 10$ \\
\hline Test 2.10 & 1D upstream (approx), on axis & 3D downstream, on axis & $\begin{array}{l}\text { 3D downstream, D/2 off } \\
\text { axis (SW) }\end{array}$ & $11: 13: 22$ & $11: 43: 42$ & $00: 30$ \\
\hline Test 2.11 & 1D upstream (approx), on axis & 3D downstream, on axis & $\begin{array}{l}\text { 3D downstream, D/2 off } \\
\text { axis (NE) }\end{array}$ & $11: 46: 31$ & $12: 23: 12$ & $00: 36$ \\
\hline
\end{tabular}

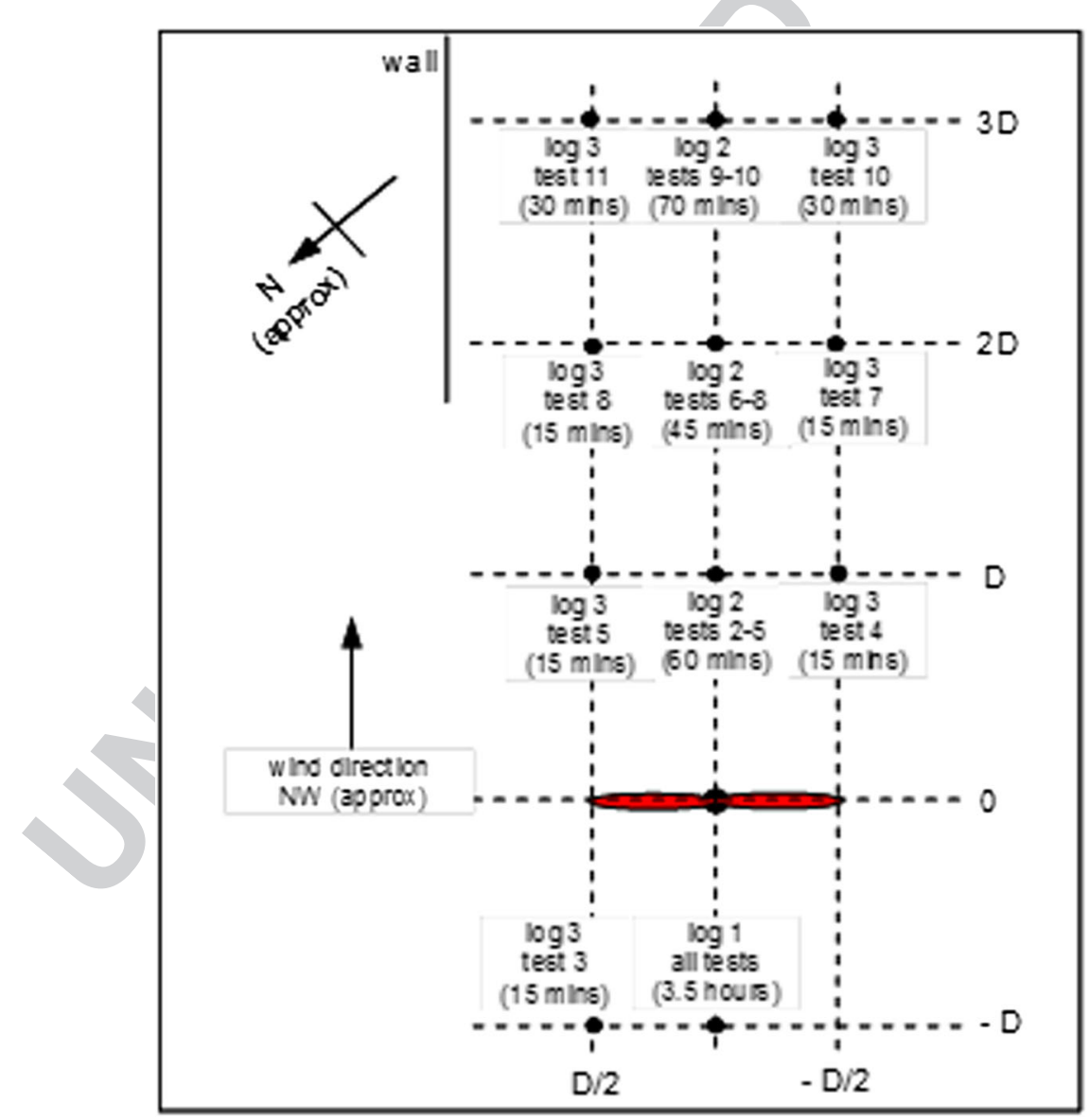

Figure 12. Pictorial representation of the test layout (session 1, day 2).

\subsubsection{Test set-up: session 2}

During session 2 recording the anemometry was set up in a different configuration, as described in Table V andT5 illustrated in Figure 13. Notably, the two downstream anemometers were now mounted at different heights $(5$ and $6 \mathrm{~m}) \mathbf{F 1 3}$ 
Table V. Anemometer position and timings for each test (session 2).

\begin{tabular}{llc}
\hline & Anemometer $1(1 \mathrm{~Hz}, 6 \mathrm{~m})$ & Anemometers $2(1 \mathrm{~Hz}, 5 \mathrm{~m})$ and $3(4 \mathrm{~Hz}, 6 \mathrm{~m})$ \\
\hline Test 4.1 & 1D upstream (approx), on axis & 1D downstream, on axis $300^{\circ}(\mathrm{WNW})$ \\
Test 4.2 & Ditto & 3D/2 downstream, on axis $320^{\circ}(\mathrm{NW})$ \\
Test 4.3 & Ditto & 2D downstream, on axis $320^{\circ}(\mathrm{NW})$ \\
Test 4.4 & Ditto & 5D/2 downstream, on axis $240^{\circ}(\mathrm{WSW})$ \\
Test 4.3A & Ditto & 2D downstream, on axis $240^{\circ}(\mathrm{WSW})$ \\
Test 4.5 & Ditto & D/2 downstream, on axis $240^{\circ}(\mathrm{WSW})$ \\
\hline
\end{tabular}

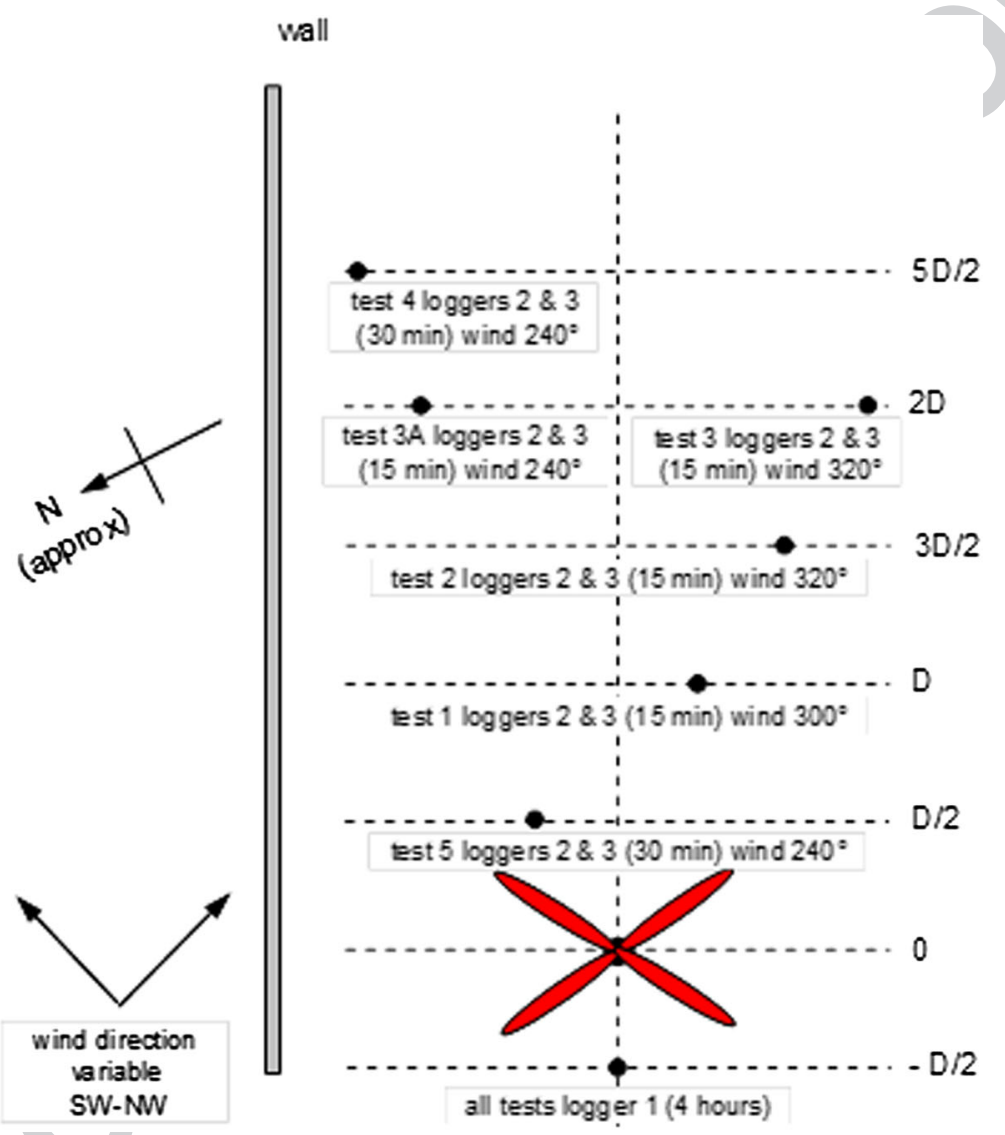

Figure 13. Pictorial representation of the test layout (session 2).

at the same location rather than at the same height but different locations (which was the set up for session 1). Also, the $6 \mathrm{~m}$ anemometer was now able to record data four times per second rather than just once per second.

\subsection{Data analysis}

\subsubsection{Analysis: session 1}

Mean wind speed, $\bar{U}\left(\mathrm{~m} \mathrm{~s}^{-1}\right)$, and standard deviation, $\sigma$, parameters were extracted from each session 1 test data set allowing turbulence intensity, I, values to be derived at each test point from equation (8).

$$
I=\frac{\sigma}{\bar{U}}
$$

The calculated turbulence intensities for day 2 are shown in Table VI, and the corresponding mean wind speeds andT6 mean wind directions are shown in Tables VII and VIII, respectively. In these tables, (L) and (R) denote left and rightT7 T8 of the centreline looking downstream from the turbine. 
Table VI. Turbulence intensity at each anemometer position (day 2).

\begin{tabular}{|c|c|c|c|c|c|c|c|c|c|c|c|}
\hline \multirow{2}{*}{$\begin{array}{l}\text { Turbulence } \\
\text { intensity }\end{array}$} & \multicolumn{11}{|c|}{ Turbulence intensity } \\
\hline & Ref $1 \mathrm{D} u / \mathrm{s}$ & Ref 1D u/s (L) & $1 \mathrm{D}$ & $1 \mathrm{D}(\mathrm{R})$ & $1 \mathrm{D}(\mathrm{L})$ & $2 \mathrm{D}$ & $2 \mathrm{D}(\mathrm{R})$ & $2 \mathrm{D}(\mathrm{L})$ & $3 \mathrm{D}$ & $3 \mathrm{D}(\mathrm{R})$ & $3 D(L)$ \\
\hline 2.1 & $30.69 \%$ & & & & & & & & & & \\
\hline 2.2 & $21.03 \%$ & & $21.09 \%$ & & & & & & & & \\
\hline 2.3 & $14.63 \%$ & $14.00 \%$ & $14.06 \%$ & & & & & & & & \\
\hline 2.4 & $16.53 \%$ & & $14.83 \%$ & $14.28 \%$ & & & & & & & \\
\hline 2.5 & $22.28 \%$ & & $24.95 \%$ & & $22.91 \%$ & & & & & & \\
\hline 2.6 & $21.30 \%$ & & & & & $22.22 \%$ & & & & & \\
\hline 2.7 & $20.34 \%$ & & & & & $18.95 \%$ & $17.69 \%$ & & & $\gamma$ & \\
\hline 2.8 & $19.48 \%$ & & & & & $23.33 \%$ & & $21.13 \%$ & & & \\
\hline 2.9 & $23.07 \%$ & & & & & & & & $24.58 \%$ & & \\
\hline 2.10 & $27.49 \%$ & & & & & & & & $27.68 \%$ & $25.67 \%$ & \\
\hline 2.11 & $30.82 \%$ & & & & & & & & $30.28 \%$ & & $29.20 \%$ \\
\hline
\end{tabular}

Table VII. Mean wind speed at each anemometer position (day 2).

\begin{tabular}{|c|c|c|c|c|c|c|c|c|c|c|c|}
\hline \multirow[b]{2}{*}{ test } & \multicolumn{11}{|c|}{ Mean wind speed $\left(\mathrm{ms}^{-1}\right)$} \\
\hline & Ref 1D us ${ }^{-1}$ & Ref 1D us ${ }^{-1}(L)$ & $1 \mathrm{D}$ & $1 \mathrm{D}(\mathrm{R})$ & $1 \mathrm{D}(\mathrm{L})$ & $2 \mathrm{D}$ & $2 \mathrm{D}(\mathrm{R})$ & $2 \mathrm{D}(\mathrm{L})$ & $3 \mathrm{D}$ & $3 D(R)$ & $3 \mathrm{D}(\mathrm{L})$ \\
\hline 2.1 & 4.01 & & & & & & & & & & \\
\hline 2.2 & 4.92 & & 5.18 & & & & & & & & \\
\hline 2.3 & 5.02 & 5.02 & 5.18 & & & & & & & & \\
\hline 2.4 & 5.01 & & 5.67 & 0.44 & & & & & & & \\
\hline 2.5 & 3.79 & & 3.89 & & 4.44 & & & & & & \\
\hline 2.6 & 4.16 & & & & & 3.83 & & & & & \\
\hline 2.7 & 4.30 & & & & & 3.87 & 4.18 & & & & \\
\hline 2.8 & 3.90 & & & & & 3.87 & & 4.12 & & & \\
\hline 2.9 & 3.44 & & & & & & & & 3.48 & & \\
\hline 2.10 & 3.30 & & & & & & & & 3.37 & 3.53 & \\
\hline 2.11 & 2.97 & & & & & & & & 2.94 & & 2.94 \\
\hline
\end{tabular}

Table VIII. Mean wind direction at each anemometer position (day 2).

\begin{tabular}{|c|c|c|c|c|c|c|c|c|c|c|c|}
\hline \multirow[b]{2}{*}{ test } & \multicolumn{11}{|c|}{ Mean wind direction $\left({ }^{\circ}\right)$} \\
\hline & $\operatorname{Ref} 1 \mathrm{D} \mathrm{us}^{-1}$ & $\operatorname{Ref} 1 \mathrm{D} \mathrm{us}^{-1}(\mathrm{~L})$ & 1D & $1 \mathrm{D}(\mathrm{R})$ & $1 \mathrm{D}(\mathrm{L})$ & $2 \mathrm{D}$ & $2 \mathrm{D}(\mathrm{R})$ & $2 \mathrm{D}(\mathrm{L})$ & $3 \mathrm{D}$ & $3 \mathrm{D}(\mathrm{R})$ & $3 D(L)$ \\
\hline 2.1 & 326.83 & & & & & & & & & & \\
\hline 2.2 & 332.37 & & 329.20 & & & & & & & & \\
\hline 2.3 & 340.64 & 316.10 & 336.60 & & & & & & & & \\
\hline 2.4 & 325.97 & > & 324.33 & 297.96 & & & & & & & \\
\hline 2.5 & 327.51 & & 326.17 & & 307.70 & & & & & & \\
\hline 2.6 & 324.23 & & & & & 302.69 & & & & & \\
\hline 2.7 & 321.17 & & & & & 298.77 & 298.36 & & & & \\
\hline 2.8 & 329.44 & & & & & 308.08 & & 308.28 & & & \\
\hline 2.9 & 316.71 & & & & & & & & 288.18 & & \\
\hline 2.10 & 319.39 & & & & & & & & 289.10 & 299.10 & \\
\hline 2.11 & 326.34 & & & & & & & & 296.31 & & 300.21 \\
\hline
\end{tabular}

The results over the 3 days showed no clear indication of any patterns of reduced velocity or increased turbulence intensity between the upstream reference values and any of the downstream locations.

To detect if any wake effects are present, one can compare the power spectral density plots of the reference wind data with those of the downstream data. An example is given in Figure 14, and it is clear that no distinguishable features areF14 present: the same was true across all the session 1 tests. This is perhaps not surprising, given the very low sampling frequency of $1 \mathrm{~Hz}$ used during these tests: any features that one might expect to see from a wake, such as the power oscillation 


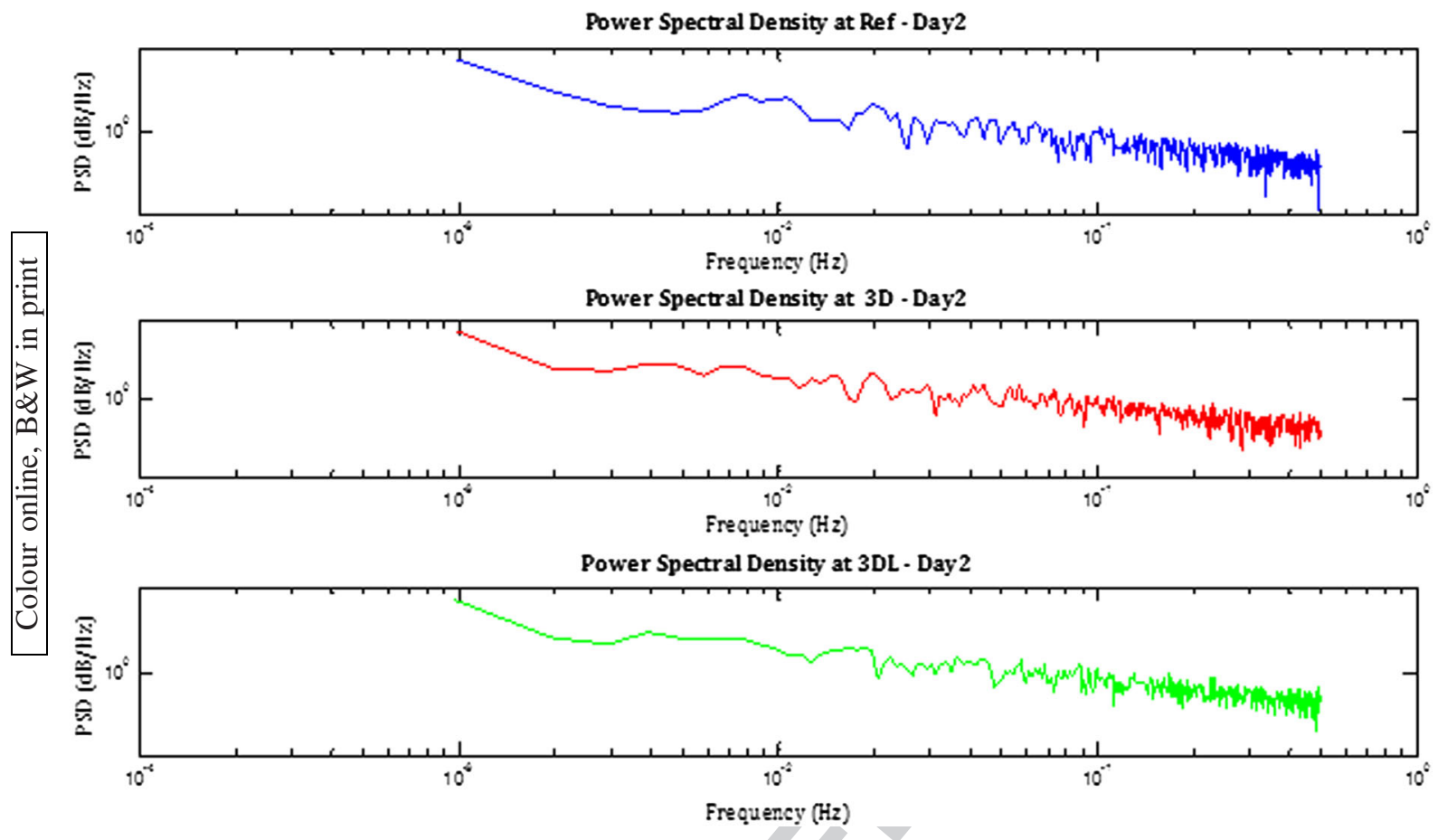

Figure 14. Power spectral density plots for test 2.11

known as $3 \mathrm{P},{ }^{24}$ caused by tower shadow effects on a three-bladed turbine occurring at a frequency of three times the blade speed (i.e. typically around $1 \mathrm{~Hz}$ ), will not be detected at this sampling frequency.

As well as the aforementioned analysis, a moving average function was implemented to investigate the nature of the wind at the test site. One-minute averages calculated every $10 \mathrm{~s}$ were plotted; an example is given in Figure 15 . Even whenF15 the large second-to-second deviations are filtered out, the plot still shows that the mean wind velocity keeps changing and does not settle around a single value for any extended periods. This confirms the assertion made previously that conditions at such a site are not highly conducive to aeolian vibrations.

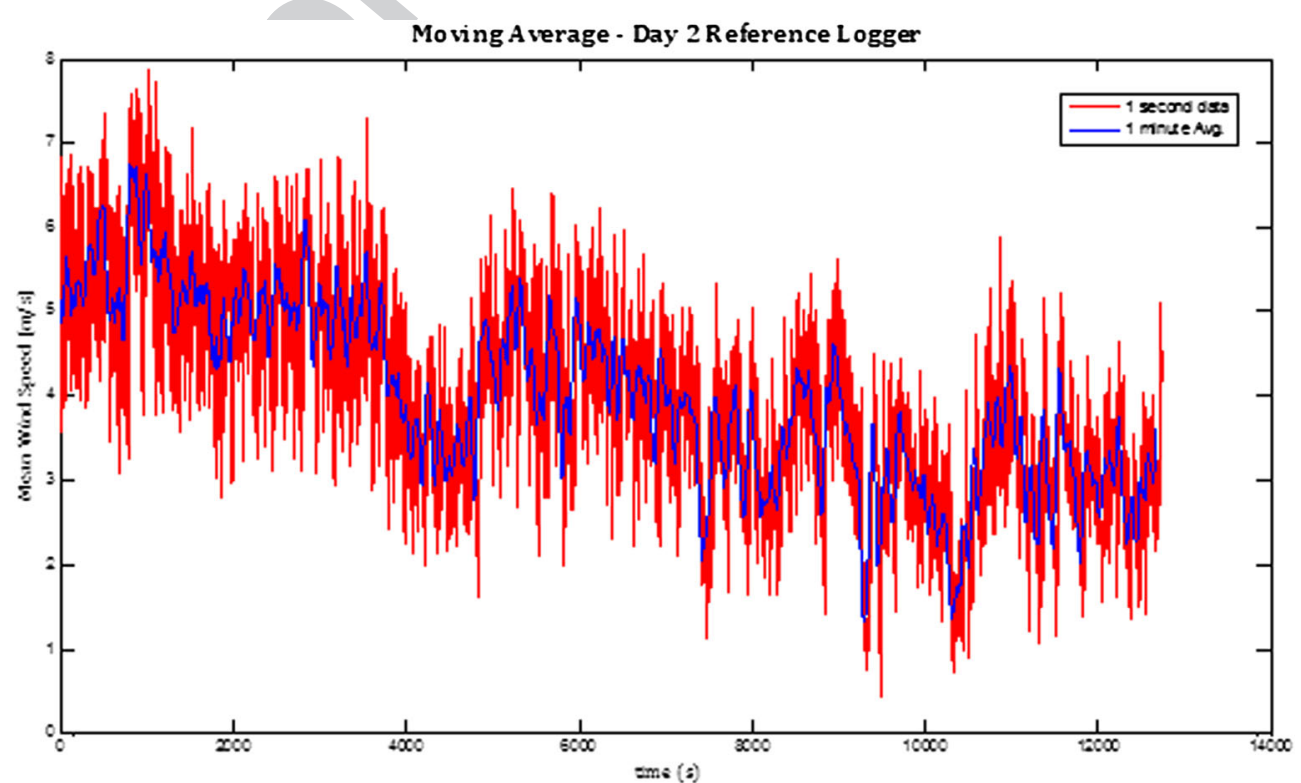

Figure 15. Moving average plot of reference anemometer (day 2). 


\subsubsection{Analysis: session 2}

The results of similar turbulence intensity calculations for the session 2 tests, along with mean wind speeds and mean wind directions, are given in Tables IX-XI, respectively.

From Table IX, the turbulence intensity appears to vary with both distance downstream and wind speed. Literature would suggest that increased turbulence intensity is likely to be a feature of the wake field for distances well downstream of the turbine, and tests 4.4 and 4.3A seem to support this assertion. Test 4.3 shows a decrease in turbulence, but this is probably due to the drop in wind speed. Regressions of turbulence intensity against distance and wind speed separately suggest that the correlation with wind speed is significant at the $90 \%$ confidence level $(P=0.07)$ whereas that with distance is not $(P=0.18)$.

From Table $X$, there is no consistent drop in mean wind speed from the reference upstream anemometer to those downstream, contrary to what might be expected, with the data again giving variable results from test to test. The wind speed does increase with height in all cases, albeit rather inconsistently, as would be expected.

The mean wind direction, Table XI, can be used to verify the validity of observations made about mean wind speed and turbulence intensity, as strong correlation between wind direction upstream and downstream of the turbine would indicate that roughly the same portion of the wind field is being observed by each anemometer. Tests 4.1 and 4.5 show good correlation of wind direction across the anemometers, which may be the result of relatively high wind speeds for these data sets. Unfortunately, these tests were relatively close to the turbine and so may miss any wake effects expected from a fully expanded wake further downstream. The low wind speeds of tests 4.3 and 4.4 lead to poor correlation in wind direction, meaning the anemometers upstream of the turbine are likely being subjected to a different portion of the wind field than the reference anemometer, making general conclusions difficult to support from this data.

Table IX. Turbulence intensity at each anemometer position (session 2).

\begin{tabular}{|c|c|c|c|c|c|c|c|c|c|c|c|}
\hline \multirow[b]{2}{*}{ Test } & \multicolumn{11}{|c|}{ Turbulence intensity } \\
\hline & Ref 1D us ${ }^{-1}$ & $\mathrm{D} / 2(5 \mathrm{~m})$ & $\mathrm{D} / 2(6 \mathrm{~m})$ & $D(5 \mathrm{~m})$ & $\mathrm{D}(6 \mathrm{~m})$ & $3 \mathrm{D} / 2(5 \mathrm{~m})$ & 3D/2 (6 m) & $2 \mathrm{D}(5 \mathrm{~m})$ & $2 \mathrm{D}(6 \mathrm{~m})$ & $5 \mathrm{D} / 2(5 \mathrm{~m})$ & $5 \mathrm{D} / 2(6 \mathrm{~m})$ \\
\hline 4.1 & $22.10 \%$ & & & $17.43 \%$ & & & & & & & \\
\hline 4.2 & $11.92 \%$ & & & & & $17.21 \%$ & $17.74 \%$ & & & & \\
\hline 4.3 & $26.85 \%$ & & & & & & & $25.49 \%$ & $24.89 \%$ & & \\
\hline 4.4 & $18.53 \%$ & & & & & & & & & $21.36 \%$ & $20.11 \%$ \\
\hline $4.3 \mathrm{~A}$ & $21.20 \%$ & & & & & & & $27.71 \%$ & $27.25 \%$ & & \\
\hline 4.5 & $19.29 \%$ & $16.34 \%$ & $16.90 \%$ & & & & & & & & \\
\hline
\end{tabular}

Table X. Mean wind speed at each anemometer position (session 2).

\begin{tabular}{|c|c|c|c|c|c|c|c|c|c|c|c|}
\hline \multirow[b]{2}{*}{ Test } & \multicolumn{11}{|c|}{ Mean wind speed } \\
\hline & Ref 1D us ${ }^{-1}$ & $\mathrm{D} / 2(5 \mathrm{~m})$ & $D / 2(6 \mathrm{~m})$ & $D(5 m)$ & $D(6 \mathrm{~m})$ & $3 \mathrm{D} / 2(5 \mathrm{~m})$ & $3 \mathrm{D} / 2(6 \mathrm{~m})$ & $2 \mathrm{D}(5 \mathrm{~m})$ & $2 \mathrm{D}(6 \mathrm{~m})$ & $5 \mathrm{D} / 2(5 \mathrm{~m})$ & $5 \mathrm{D} / 2(6 \mathrm{~m})$ \\
\hline 4.1 & 4.48 & & & 4.96 & 5.35 & & & & & & \\
\hline 4.2 & 5.24 & & & & & 4.32 & 4.73 & & & & \\
\hline 4.3 & 1.67 & & & & & & & 1.64 & 1.66 & & \\
\hline 4.4 & 2.43 & , & & & & & & & & 2.23 & 2.26 \\
\hline $4.3 \mathrm{~A}$ & 3.09 & & & & & & & 2.87 & 2.95 & & \\
\hline 4.5 & 4.67 & 5.09 & 5.16 & & & & & & & & \\
\hline
\end{tabular}

Table XI. Mean wind direction at each anemometer position (session 2).

\begin{tabular}{|c|c|c|c|c|c|c|c|c|c|c|c|}
\hline \multirow[b]{2}{*}{ Test } & \multicolumn{11}{|c|}{ Mean Wind Direction } \\
\hline & Ref 1D us ${ }^{-1}$ & $\mathrm{D} / 2(5 \mathrm{~m})$ & $\mathrm{D} / 2(6 \mathrm{~m})$ & $D(5 \mathrm{~m})$ & $D(6 m)$ & $3 D / 2(5 \mathrm{~m})$ & $3 \mathrm{D} / 2(6 \mathrm{~m})$ & 2D (5 m) & $2 \mathrm{D}(6 \mathrm{~m})$ & $5 \mathrm{D} / 2(5 \mathrm{~m})$ & $5 \mathrm{D} / 2(6 \mathrm{~m})$ \\
\hline 4.1 & 309.25 & & & 309.94 & 308.86 & & & & & & \\
\hline 4.2 & 315.15 & & & & & 304.36 & 304.59 & & & & \\
\hline 4.3 & 244.37 & & & & & & & 261.82 & 260.80 & & \\
\hline 4.4 & 221.40 & & & & & & & & & 239.69 & 237.29 \\
\hline $4.3 \mathrm{~A}$ & 224.75 & & & & & & & 229.49 & 227.25 & & \\
\hline 4.5 & 217.96 & 225.96 & 222.91 & & & & & & & & \\
\hline
\end{tabular}


Power spectra for all six tests in session 2 are plotted for the $4 \mathrm{~Hz}$ data (logger 3 ) in Figure 16. No discernible traces of enhanced frequency content in the 1-2 Hz range are detected except for a visible spike at around $1.9 \mathrm{~Hz}$ in test 4.2 (top right trace). Unfortunately, this test contained a large data gap, and so, the results presented are from a very small sample and hence must be treated with caution.

\subsection{Reliability of data}

The fact that no discernible trace of wake effects were apparent in any of the data could be considered as an affirmation of the wake analysis in Section 3, which predicted that this would likely be the case. As a check of the data processing and analysis techniques employed, an artificial periodic gust of just $0.05 \mathrm{~m} \mathrm{~s}^{-1}$ at $2 \mathrm{~Hz}$ was introduced to the data from test 4.3A, and the spectrum was again plotted as shown in Figure 17. As expected, the strong periodicity of this artificial addition is manifested as a clear increase in the spectrum at the chosen frequency.

The fact that little evidence of such phenomena (which are unlikely to manifest themselves so clearly in reality) is found in the real spectra does not conclusively prove that there are no periodic effects present in the wind field. Much of the test data was unfortunately recorded during times of very low wind speeds, and when the winds were fairly strong, Murphy's law dictated that the tests then being performed were the ones recorded at close proximity to the turbine, where any wake effects were likely to have been closer to the minimum blade height of $13 \mathrm{~m}$ than the test apparatus at 5 and $6 \mathrm{~m}$.

However, following the tests undertaken in both sessions 1 and 2, it is clear that one outcome of this work is to recommend that further testing be undertaken employing a more robust experimental set up. The most obvious change would be of the positioning of the anemometers. Although the tests were carried out to monitor impact at OHL height, it has been established that over the first 3D downstream, the wind turbine wake is unlikely to have expanded to this height ( $\sim 6 \mathrm{~m}$ above ground level). An improvement to the test set-up would therefore be to take concurrent measurements at each location, one at a height where wake effects are expected to be present and the other at $6 \mathrm{~m}$. Comparisons could then be made, which may better rule out the presence of wake effects at $6 \mathrm{~m}$. The tests in this work were limited (by site access) to a maximum distance downstream from the test turbine of $3 \mathrm{D}$. It would be very useful to increase this distance in future tests. It has been identified in Section 4 that some wake effects could be present at wood-pole height well beyond 3D downstream, and extended measurements may be able to verify this.

The low-sampling frequency of $1 \mathrm{~Hz}$ during the session 1 tests also affected the data analysis. Recognizing Nyquist sampling criterion, recording data samples at $4 \mathrm{~Hz}$ would allow examination of frequency content up to $2 \mathrm{~Hz}$. This may be enough to pick up any $3 \mathrm{P}$ wake content discussed previously, which would be expected to lie between $1-2 \mathrm{~Hz}$ for a given turbine. Only the anemometer denoted logger 3 in session 2 was capable of sampling at $4 \mathrm{~Hz}$, and ideally, all future anemometers/recorders would be capable of this data rate to pick up the expected frequency content of a wind turbine wake.
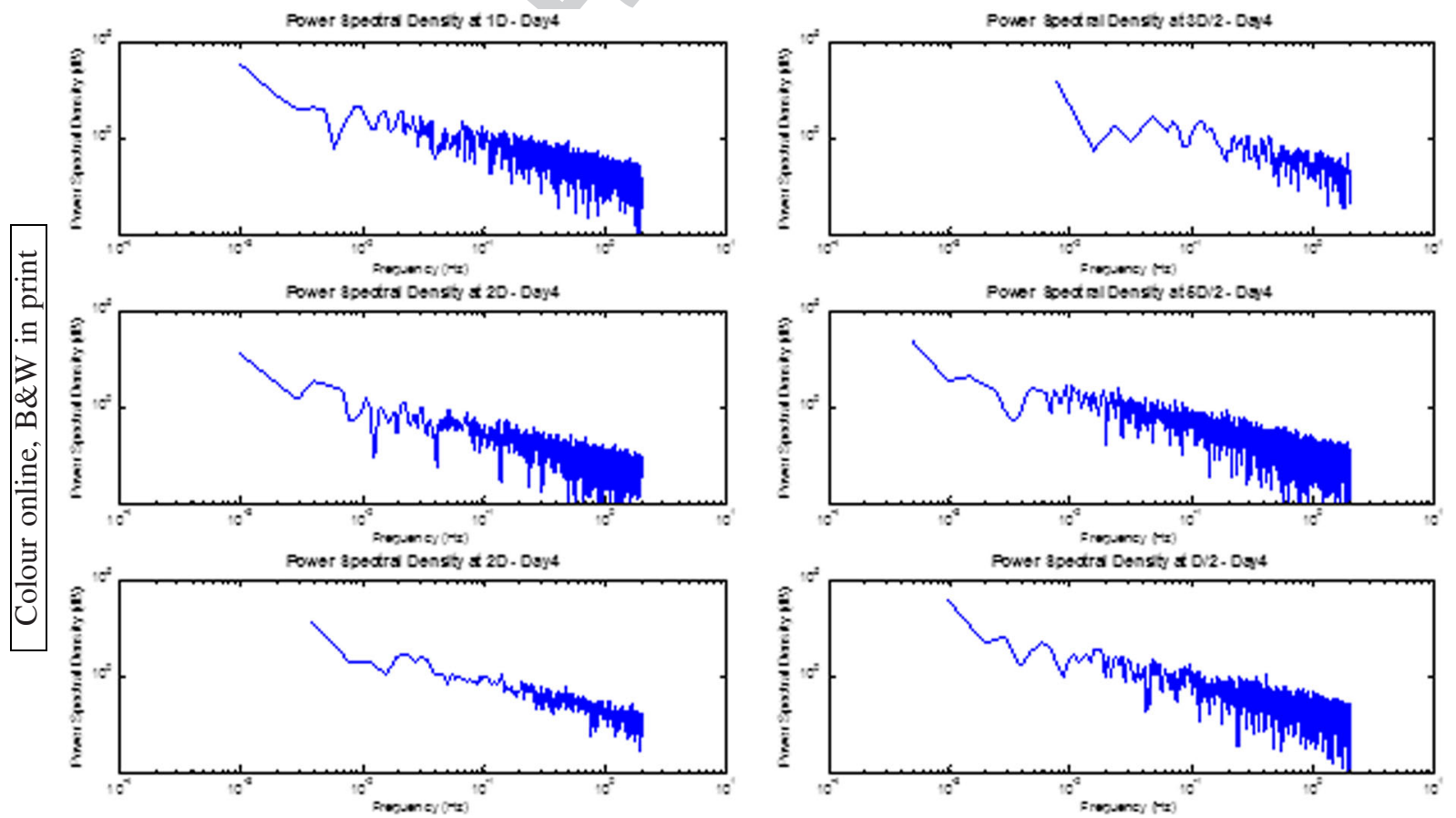

Figure 16. Power spectral density plots for tests 4.1 (top left)-4.5 (bottom right) (from Table IV and Figure 13). 

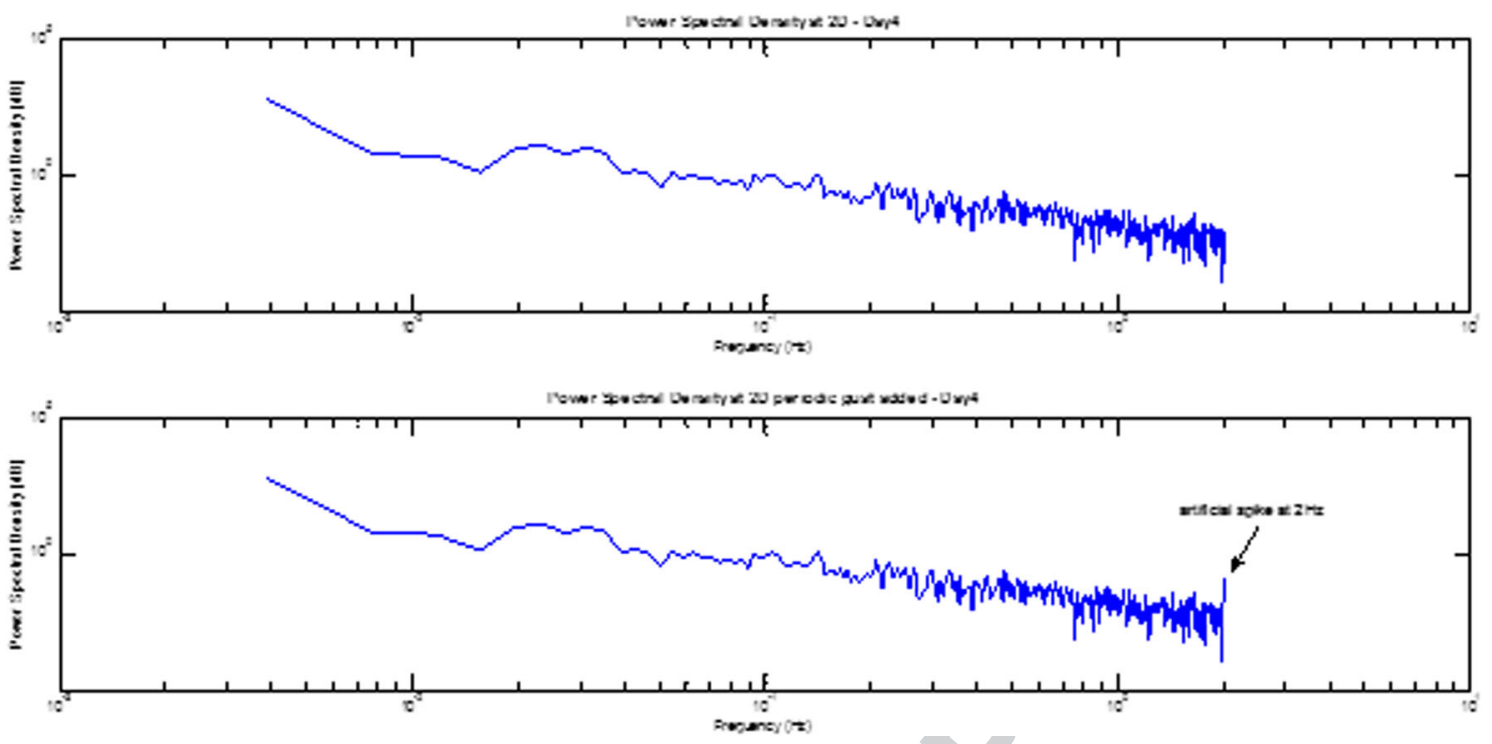

Figure 17. Power spectral density plots with artificial frequency component added.

A further issue is that on the first and third days of testing during session 1, the wind was coming from a direction such that other turbines within the farm could have been interfering with the logged data. This left only the second day of this data set as potentially reliable. Day 2 data however was characterized by low wind speeds with an average as low as $3 \mathrm{~m} \mathrm{~s}^{-1}$ for much of the day. This in itself is enough to skew turbulence intensity data as at these low wind speeds, the standard deviation is likely to be proportionally higher compared with the mean wind speed, leading to inflated and less reliable turbulence intensity values. Thus, to make further improvements, wind direction and wind speed on a test day should be such that these issues are avoided.

\section{POTENTIAL IMPACT OF WIND TURBINE WAKES ON EACH MOTION}

It was concluded in Section 4.2, from the available data and review of previous investigations, that wake effects are likely to be relatively minor, if present at all, at wood-pole OHL height. This assertion however is based on the assumption of relatively flat land, so it is conceivable that topographic circumstance could, in a minority of cases, lead to wood-pole OHLs being subjected to the full extent of a wind turbine wake. As such, the following sections outline the potential implications of such a scenario with reference to each type of conductor motion.

\subsection{Aeolian vibration}

Having reviewed the mechanism of aeolian vibration, it is clear that conditions of low turbulence and steady incoming wind are required to produce the sustained vibrations. Unless the topography of the land is particularly smooth or allows for channelled winds, these conditions are unlikely to be found close to onshore wind farms in the UK. The phenomenon is also well known, and any lines that are put up in these areas will be subject to maximum tension limits designed to minimize its effects (Section 3). Thus, with a relative degree of confidence, it can be asserted that aeolian vibrations in terrain, such as that being investigated, will be a relatively minor issue with conditions seldom likely to be conducive to sustained vortex-induced vibration.

The addition of a wake into the incident wind field would add two main features relevant to aeolian vibration: firstly, reduced mean incident velocity; and secondly, increased turbulence intensity as compared with free stream flow. The argument could be made that for an increased proportion of time, a conductor may be subject to the low wind speeds (less than $7 \mathrm{~m} \mathrm{~s}^{-1}$ ) at which aeolian vibrations occur. However, the fact that this lower mean velocity is coupled with increased turbulence intensity is the more important observation as it has already been found that turbulence helps guard against the formation and sustainability of aeolian vibrations. It can thus be concluded that the only effect of wind turbine wakes on aeolian vibrations would be positive as the already high ambient turbulence intensity would typically be amplified by the presence of the wake. 


\subsection{Conductor galloping}

As with aeolian vibration, the two main features brought by the presence of a wake that may be relevant to conductor galloping are reduced velocity and increased turbulence. As outlined in Section 2.2, conductor galloping will only occur under very precise conditions with iced conductors. The conditions must allow for the angle of attack of the wind relative to the conductor to be inside the specific regions, if they exist, whereby the Glauert-Den Hartog criterion is satisfied, as described in Section 2.2. The wind speed and level of turbulence are both factors, among many others, which may influence whether this condition is met. As each iced conductor will display different characteristics of lift and drag, it is impossible to state whether lower wind speeds and increased turbulence intensity will have positive or negative impact on the propensity of galloping to occur. It may be just as likely that the wake characteristics move conditions away from a Den Hartog zone as into one.

It must be noted however that research carried out under galloping conditions has identified that increased turbulence can significantly increase the amplitudes of vibration of galloping events when they are present ${ }^{25}$ and therefore add to the potential damage of the event. Given that galloping events in themselves are rare and require a specific set of weather conditions, it is difficult to draw conclusions supporting the case that wind turbine wake effects would have a significant impact on either the number or severity of such events.

\subsection{Turbulent buffeting}

Increased turbulence intensity, reduced velocity and periodic vorticity components evident in wind turbine wakes may all be important influences on turbulent buffeting. Increased turbulence intensity incident over some or all of a conductor span (dependant on size and direction of wake and conductor span length) could lead to increased incidence of drag force variations and so more buffeting fatigue than for a less turbulent flow. Equation (9) by Hassan, ${ }^{26}$ which built on the empirical formula first derived by Quarton and Ainslie, ${ }^{27}$ gives a value for the maximum added turbulence, $\mathrm{I}_{+}$, in the far wake region. which can be used to calculate the maximum total turbulence intensity, $\mathrm{I}_{\text {total }}$ in the far wake, Equation (10).

$$
\begin{gathered}
I_{+}=5.7 C_{T}^{0.7} I_{o}^{0.68}\left(\frac{x}{x_{n}}\right)^{-0.96} \\
I_{\text {total }}=\sqrt{I_{o}^{2}+I_{+}^{2}}
\end{gathered}
$$

$\mathrm{C}_{\mathrm{T}}$ is the turbine's thrust coefficient, which is given by $C_{T}=4 a(1-a), \mathrm{I}_{\mathrm{o}}$ is the ambient turbulence intensity and $\mathrm{x}(\mathrm{m})$ is the distance beyond the end of the near-wake region, $x_{n}(m)$. The end of the near-wake region is calculated using the method by Vermeulen, ${ }^{28}$ which uses only the thrust coefficient and some operating parameters of the turbine. It does however require calculation of several parameters, and so, the full process has been omitted from the paper. For an example turbine, taken from the test site and assuming an axial induction factor, a, of one-third, the end of the near-wake region was predicted to be around 2.5D downstream for an ambient turbulence intensity of $15 \%$. The results of the analysis, Figure 18, show a significant increase inF18

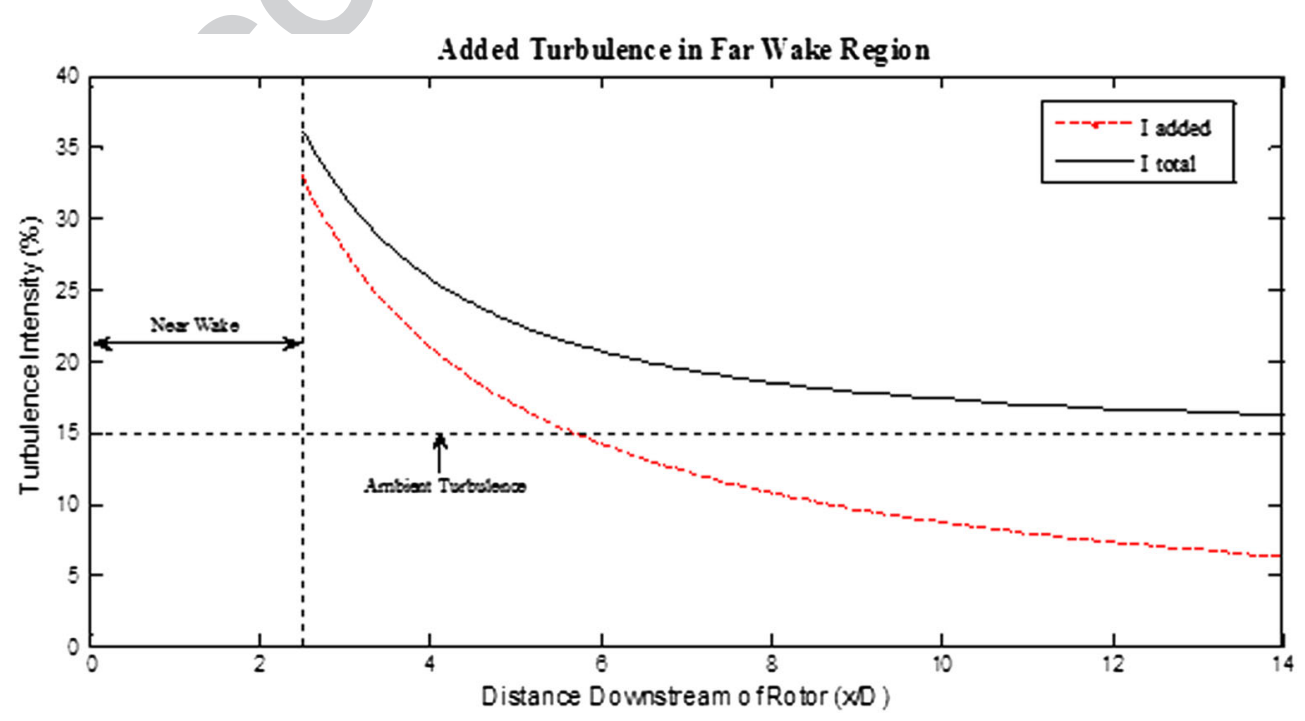

Figure 18. Added and total turbulence intensity in the far wake as function of downstream distance. 
average turbulence intensity to over $35 \%$ in the immediate far wake, more than double the original $15 \%$. This decays fairly quickly over about $4 \mathrm{D}$ to around $20 \%$ before a steadier decline back towards ambient turbulent intensity begins.

If a conductor were to lie within a wake region showing large increases in turbulence intensity, as has been demonstrated, the number of buffeting fatigue cycles it would be subject to could potentially be significantly higher than in the free stream as buffeting is inherently induced through sudden changes in the incident wind speed with respect to the conductor.

Although reduced velocity within the wake will reduce the size of the drag forces on the conductor, it would likely enhance the drag differential over the whole span as the wake would be, in most cases, only incident on part of the line. The net impact of the aforementioned two factors is a matter requiring further investigation.

Normal turbulent wind is characterized by a spectral plot with highest energy values centred on frequencies below $0.1 \mathrm{~Hz}$, as evidenced by the results from the test data site (Figure 14). Within the near wake of a wind turbine, however, the vortices shed by the blades would impart high energy content at the wind turbines 3P frequency. The 3P value of the WD34 turbines at the Coal Clough wind farm test site would be expected to fall between 1 and $2 \mathrm{~Hz}$. As such, the spectra plot of a wake, around its outer perimeter and within the first 3-4D downstream, will be characterized by a shifted spectrum with high energy content around these higher frequencies. ${ }^{20}$ As discussed in Section 3 , the natural frequency of a short-span wood-pole conductor is expected to lie anywhere between 0.3 and $0.7 \mathrm{~Hz}$, meaning that the wake spectrum could potentially excite low-order natural modes of the conductor, which could manifest, even in relatively low wind speeds, as high amplitude, low frequency oscillations and could cause similar issues to galloping in terms of mechanical loading and potential for electrical flashover.

\section{CONCLUSIONS}

This paper has investigated the potential issue of wind turbine wakes creating wind-induced motions on wood-pole OHL conductors. Such wind turbine wake-induced motions could give rise to conductor fatigue, and hence, this is an important research issue as this could impact the planning, modelling and siting of onshore wind farms. This paper has presented a literature analysis of both the mechanism by which different conductor motions are excited and the wind turbine wake characteristics having specific relevance at low levels akin to wood-pole height. An understanding of how wind turbine wakes propagate and the characteristics of the wake compared with free stream flow has been presented. The results showed little potential for wake effects at heights comparable with wood-pole lines although heightened turbulence intensity was identified as being the most likely issue. Strong effects were found to occur within the wake, in particular that the power spectral density content was altered within the near-wake region dominated by tip vortices, as illustrated in Figures 10 and 11. Large velocity deficit and high turbulence intensity features, which propagate further downstream, were also identified.

With this, and a detailed analysis of the mechanisms by which different wind-induced motions are excited, both the worst case and probable impacts of wind turbine wakes on distribution level conductor lines were presented. Issues with vortex-induced aeolian vibrations and galloping of iced conductors were found to be unlikely. Turbulent buffeting was sourced as the most concerning issue with the likelihood of increased buffeting compared with the free stream and the potential for excitation of resonant response if the conductor lies within a wake region with altered frequency content.

Uniquely, experimental data from an active wind farm, at wood-pole OHL heights, have been analysed and discussed for the first time, and the results show that wake effects at these heights are likely to be minimal.

The experimental tests, although not conclusive, have identified and clarified the testing procedure for further work on this topic, namely the use of high sampling rate anemometers $(>4 \mathrm{~Hz})$, 'long' distance measurements from the turbine (up to at least 6D from the turbine) and ideally recording data during higher mean wind speed operation. Additional investigation should also look to quantify the potential fatigue damage that could be attributable to heightened turbulent buffeting as well as investigating the potential scale of any resonant effects that may be induced. For the vast majority of wind turbine sites, wake effects will be minimal at wood-pole height, unless topography is unfavourable, and future work should aim to test a number of sites to consider the influence of topographical effects.

Lastly, the archival value of this paper is that experimental data from an active wind farm, at wood-pole OHL heights, have been analysed and discussed for the first time, and the results show that wake effects at these heights are likely to be minimal.

\section{ACKNOWLEDGEMENT}

This work was supported, via a financial contribution and the provision of experimental data, by the Strategic Technology Programme, which is managed by EA Technology Ltd. The data gathering was carried out at the Coal Clough wind farm near Burnley, Lancashire, by kind permission of Scottish Power Renewables. 


\section{REFERENCES}

1. Wareing B. Wood pole overhead lines. IEE Power and Energy Series 2005; 48. ISBN: 978-0-86341-356-8

2. National Grid. 400kV Overhead line construction, 2011. http://tinyurl.com/7hkxo5b [19 September 2012]

3. Simmonds G. Regulation of the UK electricity industry, Industry Brief, 2002. http://www.bath.ac.uk/management/cri/ pubpdf/Industry_Briefs/Electricity_Gillian_Simmonds.pdf [19 September 2012]

4. EPRI. Transmission Line Reference Book: Wind Induced Conductor Motion (2nd edn). EPRI No. No 1018554. Electric Power Research Institute: Palo Alto California, 2006.

5. ESDU. Response of structures to vortex shedding structures of circular or polygonal cross section, ESDU 96030, 1996.

6. Hardy C, Van Dyke P. Field observations on wind induced conductor motions. Journal of Fluids and Structures 1995; 9: 43-60. DOI: 10.1006/jfls.1995.1003

7. Blevins RD. Flow Induced Vibration (2nd edn). Krieger: Malabar, Florida, 2001. ISBN: 1575241838

8. Diana G, Cheli F, Fosatti F, Manetti A. Aeolian vibrations of overhead transmission lines: computation in turbulence conditions. Journal of Wind Engineering and Industrial Aerodynamics 1993; 46\&47: 639-648. DOI: 10.1016/01676105(93)90332-I

9. Cigré. SCB2 WG11 TF04: safe design tension with respect to aeolian vibration. Electra 2001; 198: $28-43$.

10. Den Hartog JP. Transmission line vibration due to sleet. AIEE Transactions 1932; 51: 1074-1076

11. Tsui YT. Modern developments in aeolian vibration. Electric Power Systems Research 1988; 15: 173-179. DOI: 10.1016/0378-7796(88)90021-1

12. Smith JW. Vibration of Structures Applications in Civil Engineering Design. Chapman and Hall, 1988. ISBN: 0412280205

13. Scottish Power Energy Networks. Specification for $11 \mathrm{kV}$ and $33 \mathrm{kV}$ overhead lines of unearthed construction on single and "H" type wood poles. OHL-03-99, Issue 1, 2006. http://www.spenergynetworks.co.uk/newconnections/pdf/OHL03-099_Issue1_ForInternet.pdf [19 September 2012]

14. CE Electric. Specification for HV wood pole lines up to and including $33 \mathrm{kV}$, NSP/004/042, 2006. http://www. northernpowergrid.com/som_download.cfm?t=media:documentmedia\&i=321\&p=file [19 September 2012]

15. Sanderse B. Aerodynamics of wind turbine wakes: literature review, ECN-e--09-016, 2009. http://www.ecn.nl/docs/ library/report/2009/e09016.pdf [19 September 2012]

16. Grant I, et al. An experimental and numerical study of the vortex filaments in the wake of an operational wind turbine. Journal of Wind Engineering and Industrial Aerodynamics 2000; 85: 177-189. DOI: 10.1016/S0167-6105(99)00139-7

17. Crespo A, Hernandez J, Frandsen S. Survey of modelling methods for wind turbine wakes and wind farms. Wind Energy 1999; 2: 1-24. DOI: 10.1002/(SICI)1099-1824(199901/03)2:1

18. Schepers JG. ENDOW: validation and improvement of ECN's wake model. Technical Report ECN-C-03-034, Energy research Centre of the Netherlands, 2003. ftp://www.nrg-nl.com/pub/www/library/report/2003/c03034.pdf [19 September 2012]

19. Ainslie JF. Calculating the flowfield in the wake of wind turbines. Journal of Wind Engineering and industrial Aerodynamics 1988; 27; 213-224. DOI: 10.1016/0167-6105(88)90037-2

20. Crespo A, Hernandez J. Turbulence characteristics in wind-turbine wakes. Journal of Wind Engineering and Industrial Aerodynamics 1996; 61: 71-85. DOI: 10.1016/0167-6105(95)00033-X

21. Smith D, Taylor GJ. Further analysis of turbine wake development and interaction data. In Proceedings of the 13th BWEA Wind Energy Conference, Swansea, UK, 1991; 325-331.

22. Troldborg N, Sorensen JN, Mikkelsen R. Numerical simulations of wake characteristics of a wind turbine in uniform flow. Wind Energy 2010; 13: 86-99. DOI: 10.1002/we.345

23. Troldborg N, Sorensen JN, Mikkelsen R. Actuator line simulation of wake of wind turbine operating in turbulent inflow. Journal of Physics: Conference Series 2007; 75: 12063. DOI: 10.1088/1742-6596/75/1/012063

24. Fox B. Wind power integration. IET Power and Energy Series 2007; 50. ISBN: 978-0-86341-449-7

25. Chadha J, Jaster W. Influence of turbulence on the galloping instability of iced conductors. IEEE Transactions on Power Apparatus and Systems 1975; PAS-94: 1489-1499.

26. Hassan U. A wind tunnel investigation of the wake structure within small wind turbine farms, E/5A/CON/5113/1890, UK Department of Energy, ETSU-WN-5113, 1992.

27. Quarton DC, Ainslie JF. Turbulence in wind turbine wakes. In Proceedings of the European Wind Energy Conference, BWEA/EWEA, Peter Peregrinus, Bristol, UK, Vol. 1, 1989; 542-546.

28. Vermeulen PEJ. An experimental analysis of wind turbine wakes. In Third International Symposium on Wind Energy Systems. BHRA, Cranfield, England, 1980; 431-450. 


\section{Author Query Form}

\section{Journal: Wind Energy}

\section{Article: we_1717}

Dear Author,

During the copyediting of your paper, the following queries arose. Please respond to these by annotating your proofs with the necessary changes/additions.

- If you intend to annotate your proof electronically, please refer to the E-annotation guidelines.

- If you intend to annotate your proof by means of hard-copy mark-up, please refer to the proof mark-up symbols guidelines. If manually writing corrections on your proof and returning it by fax, do not write too close to the edge of the paper. Please remember that illegible mark-ups may delay publication.

Whether you opt for hard-copy or electronic annotation of your proofs, we recommend that you provide additional clarification of answers to queries by entering your answers on the query sheet, in addition to the text mark-up.

\begin{tabular}{|c|l|c|}
\hline Query No. & \multicolumn{1}{|c|}{ Query } & Remark \\
\hline Q1 & $\begin{array}{l}\text { AUTHOR: Please give manufacturer information for this product } \\
\text { (Mitsubushi 300 kW turbines and Siemens turbines): company name, } \\
\text { town, state (if USA), and country. If it is USA, do not include country. }\end{array}$ & \\
\hline Q2 & $\begin{array}{l}\text { AUTHOR: "Given the findings of Section 3.1.." There is no Section } \\
\text { 3.1 heading. Please check and replace with the correct section citation. }\end{array}$ & \\
\hline Q3 & $\begin{array}{l}\text { AUTHOR: Please give address information for this manufacturer } \\
\text { (Vestas WD34 turbines): town, state (if applicable), and country. If it } \\
\text { is USA, do not include country. }\end{array}$ & \\
\hline Q4 & AUTHOR: Please define WNW. & \\
\hline
\end{tabular}


Required software to e-Annotate PDFs: Adobe Acrobat Professional or Adobe Reader (version 7.0 or above). (Note that this document uses screenshots from Adobe Reader $\mathrm{X}$ )

The latest version of Acrobat Reader can be downloaded for free at: http://get.adobe.com/uk/reader/

Once you have Acrobat Reader open on your computer, click on the Comment tab at the right of the toolbar:

닙

This will open up a panel down the right side of the document. The majority of tools you will use for annotating your proof will be in the Annotations section, pictured opposite. We've picked out some of these tools below:

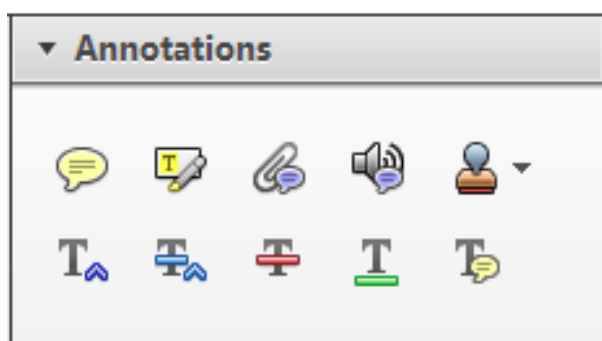

1. Replace (Ins) Tool - for replacing text.

Strikes a line through text and opens up a text box where replacement text can be entered.

How to use it

- Highlight a word or sentence.

- Click on the Replace (Ins) icon in the Annotations section.

- Type the replacement text into the blue box that appears.

Idard tramework for the analysis of $\mathrm{m}$ icy-Nevertheless, it also led to exog،

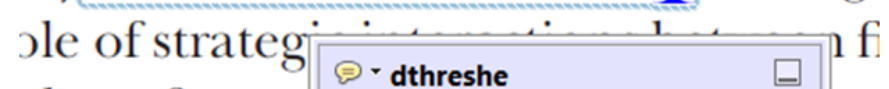
\begin{tabular}{l|l|l} 
aber of comp & 08/06/2011 15:58:17 & 0
\end{tabular} is that the $\mathrm{s} 1 \overline{\text {, which led }}$ of nain compo: be level, are exc nc

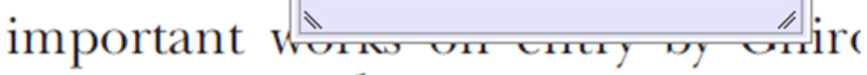
M heneferth) ${ }^{1}$ we anen the "hlarl $\mathrm{h}$

3. Add note to text Tool - for highlighting a section to be changed to bold or italic.

T Highlights text in yellow and opens up a text box where comments can be entered.

How to use it

- Highlight the relevant section of text.

- Click on the Add note to text icon in the Annotations section.

- Type instruction on what should be changed regarding the text into the yellow box that appears.

namic responses of mark ups ent with the VAR evidence

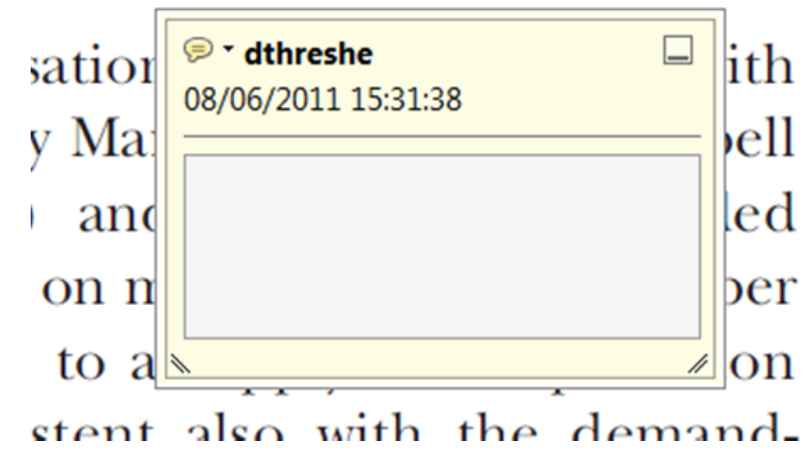

2. Strikethrough (Del) Tool - for deleting text.

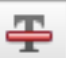

Strikes a red line through text that is to be deleted.

How to use it

- Highlight a word or sentence.

- Click on the Strikethrough (Del) icon in the Annotations section.

there is no room tor extra prohts al s ups are zero and the number of ret) values are not determined by Blanchard and Kiyotaki (1987), sfect competition in general equilil ts of aggregate demand and supply lassical framework assuming monol sen an evorenous number of firms

4. Add sticky note Tool - for making notes at specific points in the text.

Marks a point in the proof where a comment needs to be highlighted.

How to use it

- Click on the Add sticky note icon in the Annotations section.

- Click at the point in the proof where the comment should be inserted.

- Type the comment into the yellow box that appears.

iaisu airu suppiy sisuks. hivsl ui

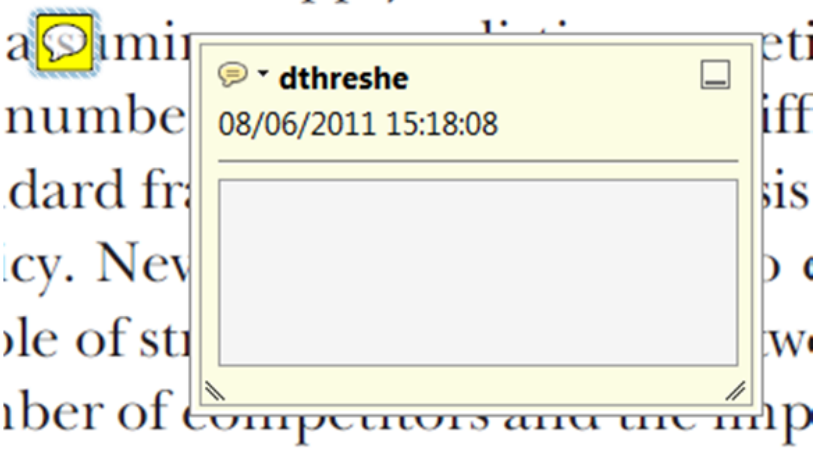

is that the structure of the sectc. 
5. Attach File Tool - for inserting large amounts of text or replacement figures.

Inserts an icon linking to the attached file in the appropriate pace in the text.

How to use it

- $\quad$ Click on the Attach File icon in the Annotations section.

- Click on the proof to where you'd like the attached file to be linked.

- Select the file to be attached from your computer or network.

- Select the colour and type of icon that will appear in the proof. Click OK.

E N D

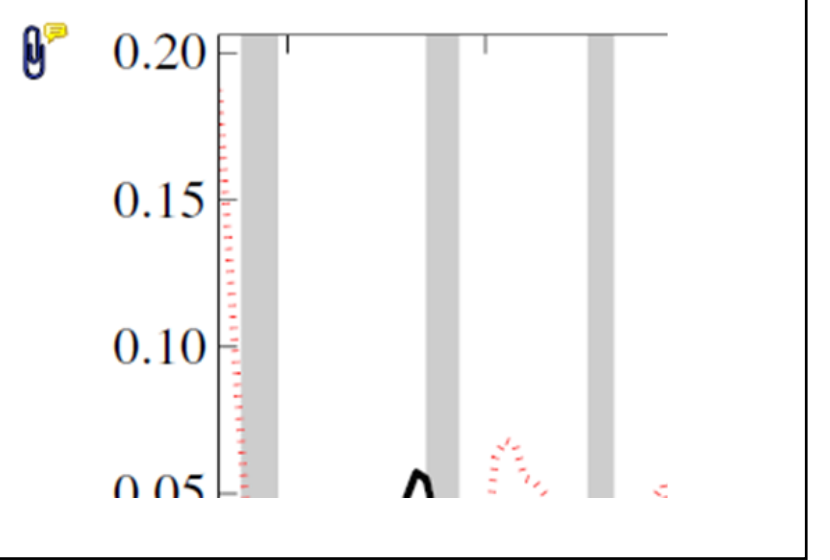

6. Add stamp Tool - for approving a proof if no corrections are required.

- Inserts a selected stamp onto an appropriate place in the proof.

\section{How to use it}

- $\quad$ Click on the Add stamp icon in the Annotations section.

- Select the stamp you want to use. (The Approved stamp is usually available directly in the menu that appears).

- Click on the proof where you'd like the stamp to appear. (Where a proof is to be approved as it is, this would normally be on the first page).

)t the Dusiness cycie, starting with the on perfect competition, constant ret

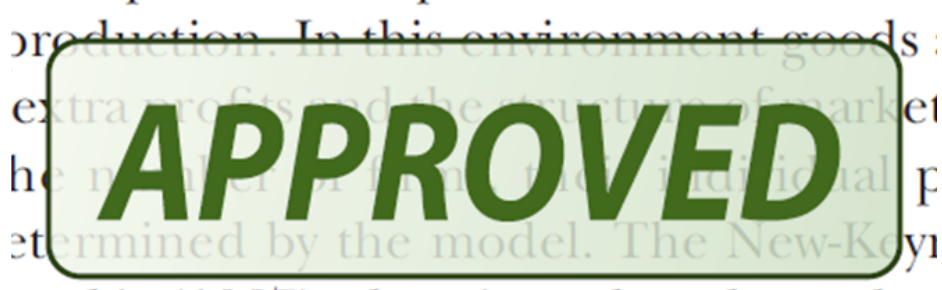
otaki (1987), has introduced produc general equilibrium models with nomin:

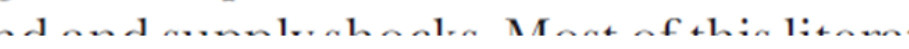

- Drawing Markups

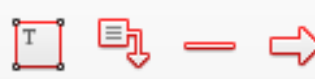

$0 \bigcirc \sqrt{6} \otimes$

\section{How to use it}

- Click on one of the shapes in the Drawing Markups section.

- Click on the proof at the relevant point and draw the selected shape with the cursor.

- To add a comment to the drawn shape, move the cursor over the shape until an arrowhead appears.

- Double click on the shape and type any text in the red box that appears.
7. Drawing Markups Tools - for drawing shapes, lines and freeform annotations on proofs and commenting on these marks.

Allows shapes, lines and freeform annotations to be drawn on proofs and for comment to be made on these marks.

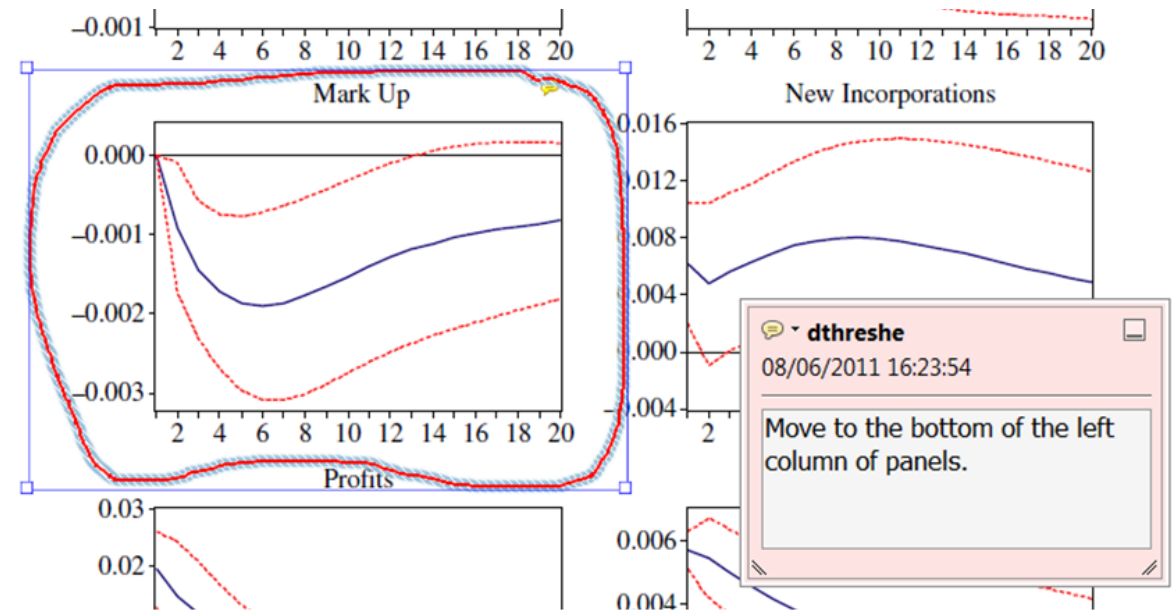

For further information on how to annotate proofs, click on the Help menu to reveal a list of further options:

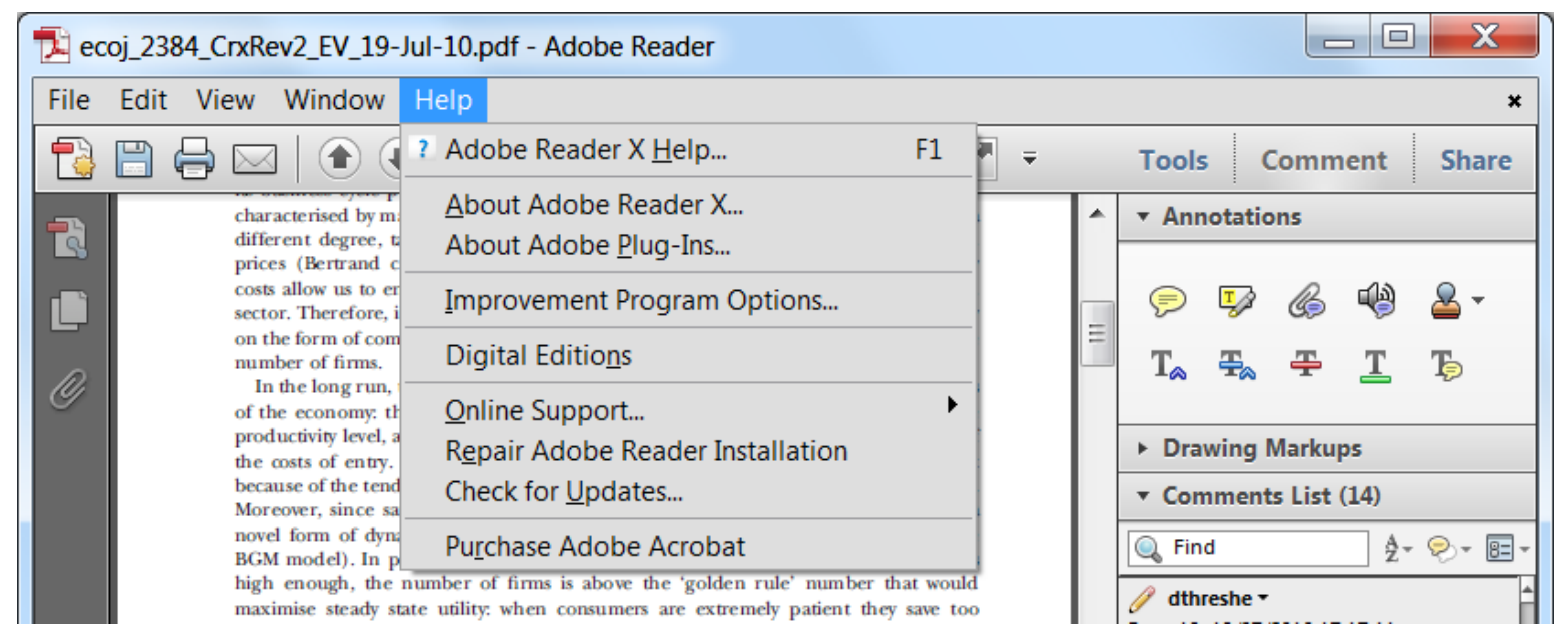

